\title{
A numerical strategy for finite element modeling of frictionless asymmetric vocal fold collision
}

Granados, Alba; Misztal, Marek Krzysztof; Brunskog, Jonas; Visseq, Vincent; Erleben, Kenny

Published in:

International Journal for Numerical Methods in Biomedical Engineering

Link to article, DOI:

$10.1002 / \mathrm{cnm} .2793$

Publication date:

2016

Document Version

Peer reviewed version

Link back to DTU Orbit

Citation (APA):

Granados, A., Misztal, M. K., Brunskog, J., Visseq, V., \& Erleben, K. (2016). A numerical strategy for finite element modeling of frictionless asymmetric vocal fold collision. International Journal for Numerical Methods in Biomedical Engineering, 33(2), [e02793]. https://doi.org/10.1002/cnm.2793

\section{General rights}

Copyright and moral rights for the publications made accessible in the public portal are retained by the authors and/or other copyright owners and it is a condition of accessing publications that users recognise and abide by the legal requirements associated with these rights.

- Users may download and print one copy of any publication from the public portal for the purpose of private study or research.

- You may not further distribute the material or use it for any profit-making activity or commercial gain

- You may freely distribute the URL identifying the publication in the public portal 


\title{
A numerical strategy for finite element modeling of frictionless asymmetric vocal fold collision
}

\author{
Alba Granados ${ }^{1 *}$, Marek Krzysztof Misztal ${ }^{2}$, Jonas Brunskog ${ }^{1}$, Vincent Visseq ${ }^{3}$ and \\ Kenny Erleben ${ }^{4}$ \\ ${ }^{1}$ Department of Electrical Engineering, Technical University of Denmark, Kgs. Lyngby DK-2800, Denmark \\ ${ }^{2}$ Niels Bohr Institute, University of Copenhagen, Copenhagen DK-2100, Denmark \\ ${ }^{3}$ Institut supérieur de mécanique de Paris, Saint-Ouen F-93400, France \\ ${ }^{4}$ Department of Computer Science, University of Copenhagen, Copenhagen DK-2100, Denmark

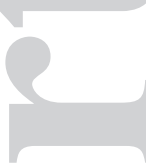

\section{SUMMARY} \\ Analysis of voice pathologies may require vocal fold models that include relevant features such as vocal \\ fold asymmetric collision. The present study numerically addresses the problem of frictionless asymmetric \\ collision in a self-sustained three-dimensional continuum model of the vocal folds. Theoretical background \\ and numerical analysis of the finite-element position-based contact model are presented, along with \\ validation. A novel contact detection mechanism capable to detect collision in asymmetric oscillations \\ is developed. The effect of inexact contact constraint enforcement on vocal fold dynamics is examined \\ by different variational methods for inequality constrained minimization problems, namely the Lagrange \\ multiplier method and the penalty method. In contrast to the penalty solution, which is related to classical \\ spring-like contact forces, numerical examples show that the parameter-independent Lagrange multiplier \\ solution is more robust and accurate in the estimation of dynamical and mechanical features at vocal \\ fold contact. Furthermore, special attention is paid to the temporal integration schemes in relation to the \\ contact problem, the results suggesting an advantage of highly diffusive schemes. Finally, vocal fold contact \\ enforcement is shown to affect asymmetric oscillations. The present model may be adapted to existing \\ vocal fold models, which may contribute to a better understanding of the effect of the non-linear contact \\ phenomenon on phonation.
}

Received.

KEY WORDS: biomechanics, vocal folds, contact detection, impact problem, variational methods, computer model.

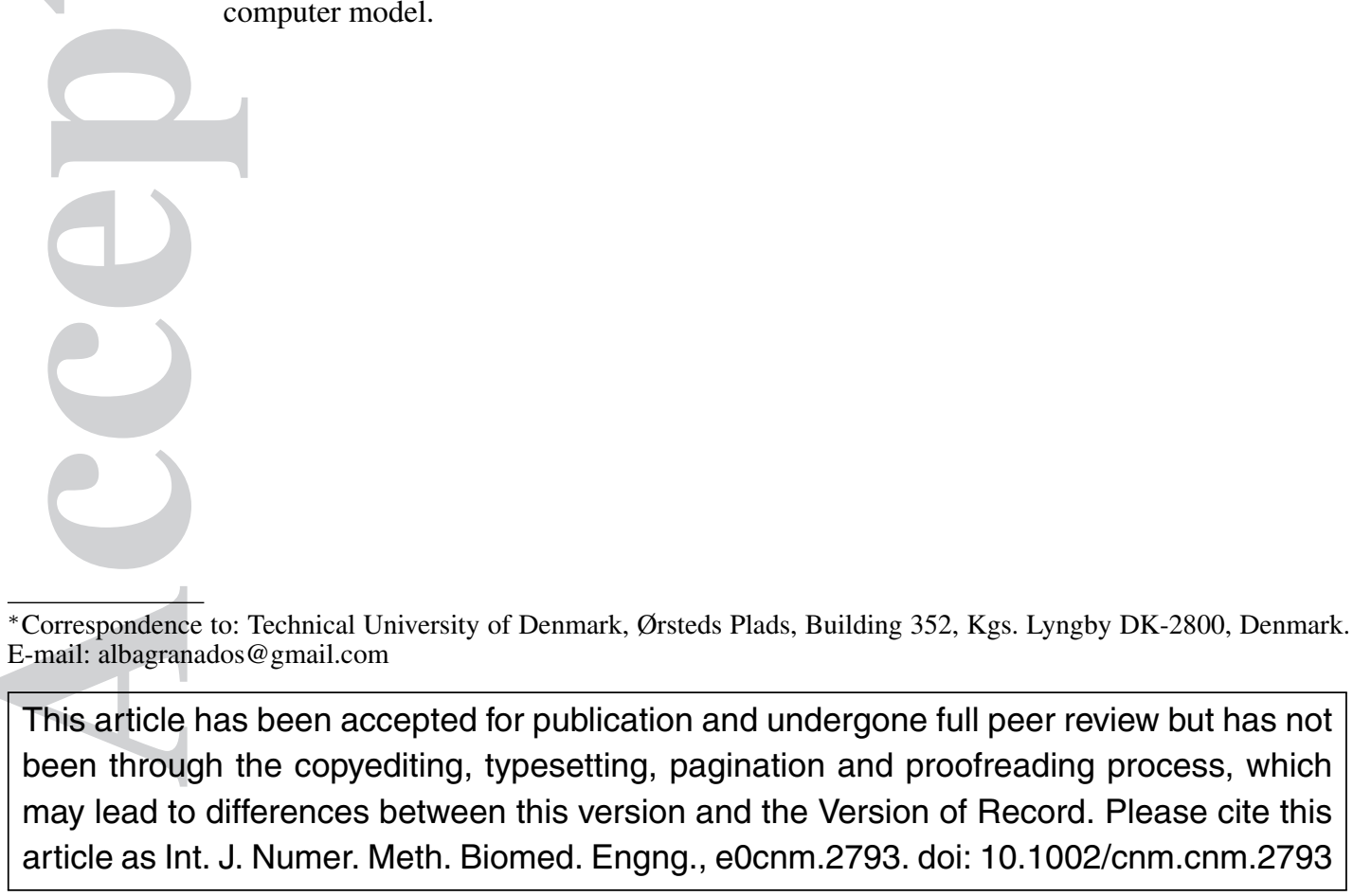




\section{INTRODUCTION}

A large number of vocal fold models have been studied in the last sixty years; from simplistic lumped-mass models driven by an incompressible Bernoulli flow [1], to continuum models coupled to advanced Navier-Stokes turbulent flows [2]. The development of vocal fold models is typically motivated by voice production or clinical research. For purposes of clinical research, studies on the mechanical and dynamical conditions that arise during vocal fold contact are of special interest, specially tissue stresses and reaction forces [3]. In that context, a three-dimensional finite element model of the vocal folds provides the framework for a detailed deformation analysis [4, 5]. In order to perform a full investigation of the vocal fold deformation, the model may include flow-structure interaction to induce vocal fold separation after contact. Furthermore, vocal fold oscillations are not generally symmetric. Therefore, allowance of asymmetric contact is of great importance.

Previous research on self-sustained vocal fold models typically include collision. In an early model presented in [6] a simple one-dimensional lumped-mass model of the vocal folds driven by an incompressible Bernoulli flow simulates collision by spring-like reaction forces. However, the simplicity of the model does result in it lacking desirable features for pathological vocal fold investigations, such as highly detailed internal stress estimations and high spatial resolution. Similar limitations may be argued in relation to the self-sustained lumped-mass model with a symmetric Hertzian contact model in [7]. In order to overcome these limitations, a finite element continuum model of the tissue is developed in [4]. The model is used to analyze the mechanics and dynamics at vocal fold closure. Symmetric vocal fold contact is assumed and the impact forces are orthogonal to a virtual rigid middle-saggital plane that prevents node interpenetration. Because airflow coupling is not included in the model, the contact phase of vocal fold separation is not investigated. Furthermore, the study limits the analysis to the mechanical output of the model, and it does not provide numerical details of the contact model. Analogous model features are found in [8], where the symmetric Hertzian contact in [7] is adapted to a continuum model of the vocal folds. Advanced models with full fluid-structure coupling typically assume symmetric vocal fold vibrations. In these cases, contact forces are modeled as spring-like forces [9], or computed by means of an Augmented Lagrangian algorithm [10], with no specific reference to contact model parameters. Furthermore, fully coupled numerical fluid-structure solvers may present topological limitations in relation to the flow solver. Hence, a minimum glottal gap is typically enforced to model the contact interaction between the two vocal folds [11], or the collision phenomenon is simply disregarded [2].

The asymmetric contact problem in self-sustained vibrations of the vocal folds is typically disregarded in continuum models of the tissue [12], or formulated in simple lumped-mass models $[6,13]$. As pointed out in [9], sophisticated contact detection algorithms are required for asymmetric oscillations and intricate vocal folds geometries, specially at the upper vocal fold edges where convex contacting surfaces are present [14]. However, a recent continuum fluid-structure model is capable of detecting asymmetric contact [15]. The contact interface constitutive equations are described on a nodal basis following a non-linear, spring-based law. A minimum glottal gap defines the contact detection zone. However, the node-to-node formulation reported in the study is not valid for general mesh generators that typically create non-matching meshes, because it does not allow for tangential and normal decomposition [16]. Furthermore, specific model contact parameters are necessary to obtain realistic vocal fold collision, but adequate experimental data is currently unavailable; this may explain the lack of validation of the vocal fold contact model in [13]. Alternatively, a more general contact model is developed in [17]. In their work, a classical combinatorial search between mesh elements at the two vocal folds is performed, despite the computational load of this operations. The contact model could potentially allow for asymmetric contact and provide detailed simulations of the impact forces. However, these features are not investigated, and the contact problem lacks validation. Furthermore, the results presented in their study seem to be inaccurate in terms of displacement, as surface contact nodes penetrate the tissue. This behavior may be a consequence of the numerical method used to enforce contact; however, the description of the numerical algorithm is missing in the study. Some vocal fold models enforce the contact constraint by the penalty method, which is equivalent to adding linear spring forces at contact $[6,9]$, or an augmented Lagrangian algorithm [10]. However, all these solution algorithms require a thoughtful choice of the model parameters, which makes the model output sensitive to arbitrariness. A recent investigation has shown that the dynamical behavior of a lumped-mass vocal fold model is highly sensitive to simplifications in the contact formulation [18]. These simplifications might lead to biased interpretation of the mechanical properties at contact, specially relevant in voice pathology 
diagnose. Finally, accuracy in contact dynamics estimation is of importance in inverse problems for vocal fold tissue parameters estimation [8].

In this paper, a three-dimensional viscoelastic continuum vocal fold model that includes a finite element model of vocal fold collision is developed. Vocal fold closure and separation is induced by an incompressible Bernoulli flow. The collision is modeled by a position-based inequality constraint, implying a constitutive contact law and a specific mechanical behavior. Different numerical procedures are adopted from contact mechanics to the vocal fold contact problem. A contact discretization technique that allows for vocal fold sliding is presented, suitable for nonmatching body meshes. Vocal fold contact is detected by a novel mechanism based on deformable interface tracking. The algorithm is capable to detect contact in asymmetric oscillations and at convex surfaces such as the upper glottal boundary, and it avoids classical combinatorial contact search. Furthermore, it is hypothesized that non-physical vocal fold interpenetration or minimum glottal gap enforcement affects the predicted dynamical and mechanical behavior of the system. Hence, the effect on vocal fold dynamics of simplifications in the contact model with regard to position-based constraints (e.g., see $[13,11]$ ) are investigated via the penalty solution algorithm. The results are compared with the proposed Lagrange multiplier formulation for exact contact constraint enforcement. Furthermore, given the high kinetic energy that the vocal folds contain just previous to collision and the short vocal fold impact time, linear spring-like contact forces are conjecture to fail at distributing the energy of impact. Hence, the penalty and the Lagrange multiplier solutions here investigated allow for a deeper understanding of the contact mechanism. Finally, the present paper investigates the effect of temporal discretization schemes on the numerical solution to vocal fold mechanics at collision.

The outline of the paper is as follows. In Section 2 the methodology to model the airflow, tissue and collision between vocal folds is presented, including numerical aspects. Section 3 is devoted to the setup for the simulation results, followed by Section 4, which includes model validation, results and discussion of the present contact model. Finally, in Section 5 the conclusions are presented.

\section{METHODOLOGY}

A three-dimensional self-sustained finite element model of the vocal folds with contact is developed. The airflow model is based on the one-dimensional models in [1, 19], and it is adapted to the threedimensional geometry of the intraglottal region. The material model is based on previous works $[5,17]$. The collision problem between the vocal folds is investigated, including mathematical formulations, the contact discretization and the collision detection mechanism. Furthermore, an analysis of the temporal discretization techniques is included to investigate the model performance at non-smooth contact.

\subsection{Glottal airflow model}

For an incompressible fluid with constant density $\rho_{\text {air }}$, the Bernoulli flow at a given time through a spatially discretized glottal canal along the longitudinal $z$-axis is described as [19]

$$
p\left(z_{i+1}\right)=p\left(z_{i}\right)+\frac{\rho_{\text {air }}}{2} U^{2} \cdot\left(\frac{1}{a\left(z_{i}\right)^{2}}-\frac{1}{a\left(z_{i+1}\right)^{2}}\right),
$$

where $U$ is the volumetric flow rate, $p\left(z_{i}\right)$ and $p\left(z_{i+1}\right)$ are aerodynamic pressures, and average flow speeds over the transverse cross-sectional areas $a\left(z_{i}\right)$ and $a\left(z_{i+1}\right)$ have been assumed; see Figure 1. Note that the aerodynamic pressures implicitly depend on time since cross-sectional areas depend on the deforming tissue boundary. No energy loss is considered neither at glottal inlet nor at glottal outlet [20]. Assuming a negligible subglottal kinetic energy, $a\left(z_{s u b}\right) \gg a\left(z_{i}\right)$, as well as no vocal tract coupling, that is, the supraglottal aerodynamic pressure is zero, from Eq. (1) the volumetric flow rate may be approximated as

$$
U=\sqrt{\frac{2 p_{\text {sub }}}{\rho_{\text {air }}}} a\left(z_{\text {det }}\right),
$$

where $p_{\text {sub }}$ is the subglottal pressure, and $a\left(z_{\text {det }}\right)$ is the cross-section where the flow detaches from the vocal folds boundaries. Previous studies have investigated flow separation in vocal folds [21]. The flow separation point is an important feature for realistic voice simulations. Since the present 
model aims at resolving vocal fold mechanics, for the sake of simplicity the flow is assumed to detach from the boundary at the minimum cross-sectional area $a\left(z_{\text {det }}\right)=a_{\min }$; downstream that point, a jet is formed, and aerodynamic forces are zero.

By spatial discretization of the vocal fold boundary, the transversal areas $a\left(z_{i}\right)$ in Eq. (1) are computed by assuming an elliptical shape, as in [17]; see Figure 1. In the present study, the computations take into account asymmetrical mediolateral deformation of the vocal folds by allowing for independent minor axis, that is,

$$
a\left(z_{i}\right)=\frac{\pi}{2} y_{i}\left(x_{i,>0}+x_{i,<0}\right),
$$

where $y_{i}$ is the major axis in the anteroposterior direction, and $x_{i,>0}$ and $x_{i,<0}$ are the positive and negative minor axes in the mediolateral direction.

\subsection{Continuum mechanical model for the vocal folds}

2.2.1. Kinematics Under deformation of a body a material particle is displaced from its original position $\mathbf{X} \in V_{\text {solid }} \subset \mathbb{R}^{3}$ (material coordinates) to its current position $\varphi(\mathbf{X})=\mathbf{x} \in v_{\text {solid }} \subset \mathbb{R}^{3}$ (spatial coordinates) via the deformation mapping $\varphi: V_{\text {solid }} \rightarrow \mathbb{R}^{3}$. The displacement field $\mathbf{u}(\mathbf{X}, t)=$ $\mathbf{X}-\mathbf{X}$ is thereby determined. From basic continuum mechanics, the motion of the body in the absence of volume forces is described by

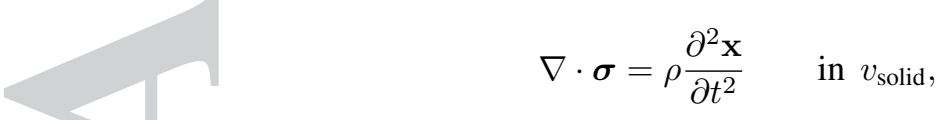

where $\rho$ is the spatial mass density, and $\sigma$ is the Cauchy stress tensor. In the case of the vocal folds, the Cauchy stress tensor at the moving airflow boundary $\Gamma_{N} \subset \partial v_{\text {solid }}$ with outward normal $\mathbf{n}$ satisfies the Neumann boundary condition

$$
\boldsymbol{\sigma} \cdot \mathbf{n}=\mathbf{p} \quad \text { on } \Gamma_{N},
$$

where $\mathbf{p}=p\left(z_{i}\right) \mathbf{n}$ from Eq. (1). Furthermore, Dirichlet boundary conditions enforce zero displacement at the anteroposterior glottal extreme regions $\Gamma_{D} \subset \partial v_{\text {solid }}$.

It is widely accepted in the voice research community, that vocal folds undertake small displacements during normal phonation [5]. In the present model, the small deformations assumption is also made, and linear theory of deformation is applied. The strain tensor is approximated by the Cauchy's strain tensor $\epsilon$. The laryngeal tissue is modeled with a compressible viscoelastic KelvinVoigt model as in [17], with dynamic viscosity $\eta$ and stiffness matrix [22]

$$
\mathcal{S}=\left(\begin{array}{cccccc}
\frac{\left(E^{\prime}-\nu^{\prime 2} E\right) E}{(1+\nu) k} & \frac{E E^{\prime} \nu^{\prime}}{k} & \frac{\left(\nu^{\prime 2} E+\nu E^{\prime}\right) E}{(1+\nu) k} & 0 & 0 & 0 \\
\frac{E E^{\prime} \nu^{\prime}}{k} & \frac{E^{\prime 2}(1-\nu)}{k} & \frac{E E^{\prime} \nu^{\prime}}{k} & 0 & 0 & 0 \\
\frac{\left(\nu^{\prime 2} E+\nu E^{\prime}\right) E}{(1+\nu) k} & \frac{E E^{\prime} \nu^{\prime}}{k} & \frac{\left(E^{\prime}-\nu^{\prime 2} E\right) E}{(1+\nu) k} & 0 & 0 & 0 \\
0 & 0 & 0 & \mu^{\prime} & 0 & 0 \\
0 & 0 & 0 & 0 & \mu^{\prime} & 0 \\
0 & 0 & 0 & 0 & 0 & \mu
\end{array}\right),
$$

where $k=E^{\prime}(1-\nu)-2 \nu^{\prime 2} E$. The constitutive equation is

$$
\boldsymbol{\sigma}(t)=\mathcal{S} \boldsymbol{\epsilon}(t)+\eta \frac{\partial}{\partial t} \boldsymbol{\epsilon}(t),
$$

where symmetry of the stress and strain tensors is assumed.

For admissible test functions $\mathbf{w}$ that vanish on $\Gamma_{D}$ with square-integrable derivatives [23], the problem takes the suitable weak form

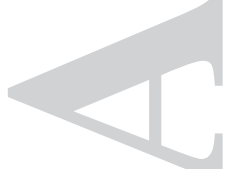

$$
\int_{v_{\text {solid }}} \rho \mathbf{w}^{T} \cdot \frac{\partial^{2} \mathbf{x}}{\partial t^{2}} \mathrm{~d} v+\int_{v_{\text {solid }}} \nabla \mathbf{w}^{T}: \boldsymbol{\sigma} \mathrm{d} v=\int_{\Gamma_{N}} \mathbf{w}^{T} \cdot \mathbf{p} \mathrm{d} \Gamma
$$

used as a starting point for the finite element formulation. In analytic mechanics, the equilibrium equations or the weak form in Eq. (8) emanate from the Hamilton's principle, that is, through the process of minimizing a functional $\Pi(\mathbf{v})$ that depends on a displacement field $\mathbf{v}$ and involves the 
potential and kinetic energies [24, 16],

$$
\Pi(\mathbf{v})=\int_{v_{\text {solid }}} \rho \mathbf{v}^{T} \frac{\partial^{2} \mathbf{v}}{\partial t^{2}} \mathrm{~d} v+\int_{v_{\text {solid }}} \nabla \mathbf{v}^{T}: \boldsymbol{\sigma} \mathrm{d} v-\int_{\Gamma_{N}} \mathbf{v}^{T} \cdot \mathbf{p} \mathrm{d} \Gamma .
$$

In this sense, Eq. (8) is called the variational equation, and $\mathbf{w}=\delta \mathbf{v}$ are the variations of the displacements or virtual displacements in the engineering literature. This study considers the vocal folds motion the solution of a minimization problem that has objective function the functional in Eq. (9).

2.2.2. Contact constraint When vocal fold collision occurs, additional boundary conditions on the contact surface $\Gamma_{C} \subset \partial v_{\text {solid }}$ arise to avoid body interpenetration, leading to a variational term $\mathrm{C}_{C}$ which must be added to the left-hand side of Eq. (8). As the large displacements take place in the mediolateral direction [17], large relative tangential movements are not expected to occur in the contact interface. Therefore, frictionless contact is assumed in this work, even though the following formulation can be easily extended to frictional contact. The non-penetration constraint may be expressed in the current configuration $v_{\text {solid }}$ in terms of the normal gap $g_{N}$ in the classical masterslave configuration as [16]

$$
g_{N}=\left(\mathbf{x}^{s}-\overline{\mathbf{x}}^{m}\right) \cdot \overline{\mathbf{n}}^{m} \geq 0 .
$$

The superscript $s$ corresponds to the interface slave node that penetrates into the master surface denoted by the superscript $m$. The vector $\overline{\mathbf{n}}^{m}$ is the outward normal on the master surface at the point $\overline{\mathbf{x}}^{m}$, which is the projection of $\mathbf{x}^{s}$ onto the master surface; see Figure 2 for an illustration. When contact occurs, that is, when $g_{N}=0$, a Cauchy traction vector $\overline{\mathbf{t}}$ is active at the contact point. This reaction force must satisfy the action-reaction principle, and for non-adhesion contact its normal component $r_{N}=\overline{\mathbf{t}} \cdot \overline{\mathbf{n}}^{m}$ must be compressive. These conditions may be written in a compact form as

$$
g_{N} \geq 0, \quad r_{N} \leq 0, \quad g_{N} r_{N}=0 \quad \text { on } \Gamma_{C},
$$

which are referred to as primal feasibility, dual feasibility and complementary slackness, respectively, in Lagrangian duality theory. Nevertheless, the classical master-slave configuration that has been described requires two implementation issues to be taken into consideration in the vocal fold problem. Firstly, collision at the glottal exit may not be detected due to the convexity of the tissue surface boundary, where non-existent or positive normal gaps may be misleading. Secondly, the geometrical and mechanical symmetry of the system makes the master and slave choice arbitrary. As arbitrariness in the master-slave configuration may have a negative effect on the solution, all slaves nodes must be tested against all surfaces, increasing thereby the computational time. In later sections, a novel detection mechanism is presented, which arises as a solution to the problems that classical master-slave implementation may cause.

The branch of mathematics of calculus of variations provides multiple methodologies to solve a constrained nonlinear minimization problem, such as the present. In this work, the Lagrange multiplier and the penalty methods based on [16] are investigated. These methods can be applied to enforce the collision constraints in Eq. (10) in an exact or a relaxed way, respectively. The Lagrange multiplier solution is here proposed as an alternative to the inexact vocal fold collision solutions typically found in the existing literature. In the next paragraphs, the new variational equations are described.

The Lagrangian approach consists of enforcing non-penetration by appending a functional

$$
\Pi(\mathbf{v}, \lambda)^{L M}=\int_{\Gamma_{C}} \lambda g_{N}(\mathbf{v}) \mathrm{d} \Gamma
$$

to the energy functional $\Pi(\mathbf{v})$ in Eq. (9), with $\lambda$ being a scalar parameter called the Lagrange multiplier or dual variable, and $g_{N}$ is the normal gap in Eq. (10). The resulting functional is called the Lagrangian function. The Lagrange multiplier can be seen as the non-positive reaction pressure $r_{N}$ necessary to avoid body interpenetration, that is, $r_{N}=\lambda<0$. Variation of the functional leads to the variational term

$$
C_{C}^{L M}=\int_{\Gamma_{C}} \lambda\left(\mathbf{w}^{s}-\overline{\mathbf{w}}^{m}\right) \cdot \overline{\mathbf{n}}^{m} \mathrm{~d} \Gamma+\int_{\Gamma_{C}} \delta \lambda\left(\mathbf{x}^{s}-\overline{\mathbf{x}}^{m}\right) \cdot \overline{\mathbf{n}}^{m} \mathrm{~d} \Gamma
$$


where $\delta \lambda$ refers to the virtual Lagrange multiplier. The above term must be combined with Eq. (8), and together with conditions in Eq. (11), they constitute the Kuhn-Tucker-Karush necessary conditions for optimality [25]. One of the drawbacks of the Lagrange method is the increase of the dimension of the system by addition of unknowns in the form of Lagrange multipliers. Furthermore, necessary conditions for the solution to be optimal lead to an indefinite system matrix, that is, critical points occur at saddle points [25].

In contrast to the exact contact boundary condition enforcement method previously described, allowance of the vocal fold interpenetration at collision is widely used in vocal fold models [6, 17, 9]. This interpenetration is a consequence of a penalty method approach to the constrained minimization problem, which corresponds to spring-like reaction force. The method consists of the approximation of the Lagrange multiplier, or reaction pressure, as $\lambda \approx \kappa g_{N}$, with $\kappa$ a positive scalar that controls how severely the constraint is violated. The energy functional related to the contact interface is

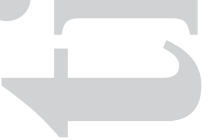

$$
\Pi(\mathbf{v})^{P}=\int_{\Gamma_{C}} \frac{1}{2} \kappa\left(g_{N}(\mathbf{v})\right)^{2} \mathrm{~d} \Gamma .
$$

Variation of this functional yields

$$
\mathbf{C}_{C}^{P}=\int_{\Gamma_{C}} \kappa\left(\mathbf{w}^{s}-\overline{\mathbf{w}}^{m}\right) \cdot\left(\mathbf{x}^{s}-\overline{\mathbf{x}}^{m}\right) \mathrm{d} \Gamma,
$$

which must be combined with Eq. (8). The penalty method maintains the dimension of the collisionfree problem. Enforcing the non-penetration constraint by increasing the penalty parameter $\kappa$ derives in an ill-conditioning of the global matrix, and collision forces with no physical sense. Therefore, the user is required to make a thoughtful choice of the penalty parameter. Numerical solutions of the Lagrange multiplier and penalty approaches for vocal fold contact are investigated in Section 4.2.

\subsection{Discretizations}

2.3.1. Spatial discretization The solid spatial discretization is performed in a simple way typically found in the literature (e.g., see [5]). Considering the spatial finite element discretization of the solid domain in $n_{e l}$ elements and $n_{n p}$ nodal points, the equilibrium Eq. (8) yields the matrix form

$$
\mathbf{M} \ddot{\mathbf{x}}+\mathbf{C} \dot{\mathbf{x}}+\mathbf{K}(\mathbf{x}-\mathbf{X})=\mathbf{F},
$$

where $\mathbf{M}, \mathbf{C}$, and $\mathbf{K}$ are the mass, damping, and stiffness $3 n_{n p} \times 3 n_{n p}$ matrices, respectively, $\mathbf{F}$ is the $3 n_{n p}$-vector of applied aerodynamic forces, and $\mathbf{x}$ and $\mathbf{X}$ are the $3 n_{n p}$-vectors that contain the current and the original positions of the nodal points, respectively; dots indicate time derivatives. By decomposition of the test functions $\mathbf{w}$ in a finite number of linear interpolation functions defined in a tetrahedral mesh, the system matrices in Eq. (16) can be easily derived by means of isoparametric transformations [23].

The aerodynamic forces in Eq. (16) are derived from the extension of the airflow spatial discretization of the one-dimensional problem in [6] to the present three-dimensional solid boundary. The linear aerodynamic forces below the flow separation point acting on the three vertices of a triangular element face $\partial v_{\text {solid }}^{e} \subset \Gamma_{N}$ with current positions $\mathbf{x}_{i}=\left(x_{i}, y_{i}, z_{i}\right), \mathbf{x}_{j}=\left(x_{j}, y_{j}, z_{j}\right)$, and $\mathbf{x}_{k}=\left(x_{k}, y_{k}, z_{k}\right)$ satisfy

$$
\mathbf{F}_{l}=-\mathbf{n} \cdot F_{l} \cdot \begin{cases}\frac{\Delta^{e}}{6}\left(p\left(z_{i}\right)+\frac{p\left(z_{j}\right)}{2}+\frac{p\left(z_{k}\right)}{2}\right) & \text { if } l=i \\ \frac{\Delta^{e}}{6}\left(\frac{p\left(z_{i}\right)}{2}+p\left(z_{j}\right)+\frac{p\left(z_{k}\right)}{2}\right) & \text { if } l=j \\ \frac{\Delta^{e}}{6}\left(\frac{p\left(z_{i}\right)}{2}+\frac{p\left(z_{j}\right)}{2}+p\left(z_{k}\right)\right) & \text { if } l=k\end{cases}
$$

where $p\left(z_{l}\right)$ correspond to the pressure derived from Eq. (1) applied to each nodal point $\mathbf{x}_{i}, \mathbf{x}_{j}$ and $\mathbf{x}_{k}$, and $\Delta^{e}$ stands for the area of the triangular element face with outward normal vector $\mathbf{n}$. Downstream the flow separation point, aerodynamic forces are zero

When collision occurs, the finite element discretization of Eq. (13) or Eq. (15) must be calculated. A node-to-surface contact element may be described by a master element surface, defined by target vertices $\mathbf{x}_{1}, \mathbf{x}_{2}$ and $\mathbf{x}_{3}$, and a contactor slave node $\mathbf{x}^{s}$, belonging to the opposite vocal fold; see Figure 2 (right). The triangular interface geometry can be parametrized by an isoparametric 
transformation with linear functions $N_{I}(\xi, \zeta)$ and local variables $(\xi, \zeta)$ defined on a reference triangular element with vertices $(0,0),(1,0)$ and $(0,1)$. Hence, the displacement constraint in Eq. (10) yields

$$
\left(\mathbf{x}^{s}-\sum_{I=1}^{3} N_{I}(\bar{\xi}, \bar{\zeta}) \mathbf{x}_{I}\right) \cdot \overline{\mathbf{n}}^{m} \geq 0,
$$

with $(\bar{\xi}, \bar{\zeta})$ satisfying

$$
\left(\mathbf{x}^{s}-\sum_{I=1}^{3} N_{I}(\bar{\xi}, \bar{\zeta}) \mathbf{x}_{I}\right) \cdot\left(\mathbf{x}_{k}-\mathbf{x}_{1}\right)=0
$$

for $k=2,3$, which corresponds to the equations to find the projection $\overline{\mathbf{x}}^{m}$ of $\mathbf{x}^{s}$ onto the master surface. Consequently, prescribed position constraints can be expressed by a contact element matrix

$$
\mathbf{g}^{e}=\left(\overline{\mathbf{n}}^{m}-N_{1}(\bar{\xi}, \bar{\zeta}) \overline{\mathbf{n}}^{m}-N_{2}(\bar{\xi}, \bar{\zeta}) \overline{\mathbf{n}}^{m}-N_{3}(\bar{\xi}, \bar{\zeta}) \overline{\mathbf{n}}^{m}\right) \in \mathbb{R}^{1 \times 12}
$$

with $\mathbf{g}^{e} \cdot\left(\mathbf{x}^{s} \mathbf{x}_{1} \mathbf{x}_{2} \mathbf{x}_{3}\right)^{T} \geq 0$. Note that for linear shape functions the outward normal is independent of the projection point. For $n_{c e}$ contact elements with negative normal gap, a finite element assembly algorithm can be used to build a global constraint contact $n_{c e} \times 3 n_{n p}$ matrix $\mathbf{G}$.

Enforcing the contact displacement constraint with the Lagrangian approach in a finite element formulation, requires the spatial discretization of the variational term in Eq. (13). Interpretation of the Lagrange multipliers as reaction forces, makes it possible to remove the surface integrals in Eq. (13). Hence, the equations that provide the current equilibrium position with $n_{c e}$ active contact elements are

$$
\begin{aligned}
\mathbf{M} \ddot{\mathbf{x}}+\mathbf{C} \dot{\mathbf{x}}+\mathbf{K}(\mathbf{x}-\mathbf{X})+\mathbf{G}^{T} \boldsymbol{\Lambda} & =\mathbf{F} \\
\mathbf{G x} & =\mathbf{0},
\end{aligned}
$$

where $\boldsymbol{\Lambda}=\left(\lambda_{1}, \cdots, \lambda_{n c}\right)^{T}$ is a $n_{n c^{-}}$-vector that contains non-positive reaction forces, and the $3 n_{n p^{-}}$ vector $\mathbf{G}^{T} \boldsymbol{\Lambda}$ contains the applied contact force at each degree of freedom under contact. For positive Lagrange multipliers, the corresponding contact element must be removed from the global constraint contact matrix to allow for contact surface separation.

By spatial finite element discretization of the variation in Eq. (15), the equilibrium position $\mathbf{x}$ that fulfills the contact constraint in a relaxed way via a penalty formulation satisfies

$$
\mathbf{M} \ddot{\mathbf{x}}+\mathbf{C} \dot{\mathbf{x}}+\mathbf{K}(\mathbf{x}-\mathbf{X})+\mathbf{G}^{T} \kappa \mathbf{G} \mathbf{x}=\mathbf{F},
$$

where the penalty parameter $\kappa$ accounts for the area of the master surfaces, so that the surface integral in Eq. (15) is neglected. The penalty term can be interpreted as a reaction force normal to the master surface and proportional to the contact element volume interpenetration. From this observation, computations can be simplified, as will be shown in Section 2.4.

Enforcement of Dirichlet boundary conditions at $n_{d}$ nodal points on $\Gamma_{D}$ may be expressed in terms of a global solid boundary $n_{d} \times 3 n_{n p}$ matrix $\mathbf{D}$ as

$$
\mathbf{D}(\mathbf{x}-\mathbf{X})=\mathbf{0} .
$$

Since the constraint matrix has to be assembled only once, the Lagrangian approach with a $n_{d}$-vector of unknowns $\boldsymbol{\Lambda}^{D}$ can be used to modify the equilibrium equation in Eq. (16) as in Eq. (21) to enforce Dirichlet boundary conditions. For equality constraints as in Eq. (23), the Lagrange multipliers can take on both positive and negative values.

2.3.2. Temporal discretization To solve for the equilibrium current position $\mathrm{x}$, attention has to be paid to temporal discretization schemes, since instabilities and accuracy problems may arise. The accuracy analysis found in most of the literature assumes smoothness of the system, as well as small Rayleigh damping [23]. However, vocal fold contact modeling results in a non-smooth system. Simple lumped-mass models of the vocal folds include a smooth contact activation function [6], which makes classical accuracy analysis of the temporal integration schemes valid. The present finite element vocal fold model is highly non-linear and non-smooth, and due to its complexity the use of smooth activation functions requires alternative contact detection mechanism. Hence, the 
effect of the temporal schemes in the present system requires computer evaluation. In this section, temporal integration algorithms and its basic features are summarized and adapted to the vocal fold contact equations.

The most common schemes to temporally discretize the hyperbolic problem belong to the Newmark family of implicit one-step methods [26], as used in [17]. These schemes consist of the equations

$$
\begin{gathered}
\mathbf{x}_{n+1}=\underbrace{\mathbf{x}_{n}+h \dot{\mathbf{x}}_{n}+\frac{h^{2}}{2}(1-2 \beta) \ddot{\mathbf{x}}_{n}}_{\tilde{\mathbf{x}}_{n+1}}+\beta h^{2} \ddot{\mathbf{x}}_{n+1}, \\
\dot{\mathbf{x}}_{n+1}=\underbrace{\dot{\mathbf{x}}_{n}+(1-\gamma) h \ddot{\mathbf{x}}_{n}}_{\tilde{\mathbf{x}}_{n+1}}+\gamma h \ddot{\mathbf{x}}_{n+1},
\end{gathered}
$$

where now the subscript $n$ indicates that the function is approximated at time $t_{n}, h$ is the time step, $\gamma$ and $\beta$ are the algorithm parameters associated to the quadrature scheme, and $\tilde{\mathbf{x}}_{n+1}$ and $\tilde{\dot{\mathbf{x}}}_{n+1}$ are the so-called predictors. The algorithm parameters control accuracy and stability of the scheme, which can be studied by spectral analysis of the amplification matrix [23]. For smooth systems, and if Rayleigh damping is assumed, the conditions $2 \beta \geq \gamma \geq \frac{1}{2}$ (if equality, it corresponds to an undamped Newmark scheme, as used in e.g., [9]) and $\beta=(\gamma+1 / 2)^{2} / 4$ with $\gamma>1 / 2$ assures unconditional stability. The latter values introduce numerical dissipation in all frequency range, specially at higher modes, where typically finite element solution seems to deteriorate. Therefore, the scheme may be considered advantageous. However, the accuracy of the algorithm drops to first order.

An alternative method with second-order accuracy and numerical damping at high frequencies is the Hilber-Hughes-Taylor $\alpha$-method [27]. The scheme consists of a weighted mean of the Newmark approximations in Eqs. (24) as $(1+\alpha) \mathbf{x}_{n+1}-\alpha \mathbf{x}_{n}$ and $(1+\alpha) \dot{\mathbf{x}}_{n+1}-\alpha \dot{\mathbf{x}}_{n}$, respectively. When $\alpha$ is zero, the scheme is equivalent to the Newmark family of methods. The weight parameter $\alpha$ controls the amount of numerical dissipation; the smaller, the wider mode damping is introduced, keeping a higher low mode damping. It has been shown that for $\alpha \in[-1 / 3,0], \gamma=0.5-\alpha$ and $\beta=(1-\alpha)^{2} / 4$, the scheme is unconditionally stable with second-order accuracy. Nevertheless, the scheme introduces a phase error larger than the one produced by an undamped Newmark rule, with increases as the parameter $\alpha$ is decreased.

In the case of the Lagrange multiplier formulation of the constrained problem, the temporal integration scheme leads to the system of linear equations

$$
\begin{gathered}
{\left[\begin{array}{ccc}
\mathbf{A} & \mathbf{D}^{T} & \mathbf{G}_{n+1}^{T} \\
(1+\alpha) h^{2} \beta \mathbf{D} & \mathbf{0} & \mathbf{0} \\
(1+\alpha) h^{2} \beta \mathbf{G}_{n+1} & \mathbf{0} & \mathbf{0}
\end{array}\right] \cdot\left[\begin{array}{c}
\ddot{\mathbf{x}}_{n+1} \\
\boldsymbol{\Lambda}_{n+1}^{D} \\
\boldsymbol{\Lambda}_{n+1}
\end{array}\right]=} \\
{\left[\begin{array}{c}
\mathbf{F}_{n}-\mathbf{C}\left((1+\alpha) \tilde{\mathbf{x}}_{n+1}-\alpha \dot{\mathbf{x}}_{n}\right)-\mathbf{K}\left((1+\alpha) \tilde{\mathbf{x}}_{n+1}-\alpha \mathbf{x}_{n}-\mathbf{X}\right) \\
-\mathbf{D}\left((1+\alpha) \tilde{\mathbf{x}}_{n+1}-\alpha \mathbf{x}_{n}-\mathbf{X}\right) \\
-\mathbf{G}_{n+1}\left((1+\alpha) \tilde{\mathbf{x}}_{n+1}-\alpha \mathbf{x}_{n}\right)
\end{array}\right],}
\end{gathered}
$$

with

$$
\mathbf{A}=\mathbf{M}+(1+\alpha) \gamma h \mathbf{C}+(1+\alpha) h^{2} \beta \mathbf{K}
$$

The global contact constraint matrix $\mathbf{G}_{n+1}$ is built from active contact elements at time $t_{n+1}$, and $\mathbf{F}_{n}$ is the vector of aerodynamic forces at time $t_{n}$. The other matrices are kept constant, since small volume element variations are assumed in relation to the small displacement assumption. In Section 4.3 a numerical study of the temporal integration schemes is presented.

\subsection{Deformable interface tracking for contact detection}

Collision detection mechanism is a non-trivial problem in transient contact mechanics. Generally based on the normal gap sign in Eq. (10), detection mechanisms require the calculation of the projection of the slave node onto the master surface. For a piecewise linear finite element surface, this projection may lead to existence or misdetection problems, especially for slave nodes situated at glottal exit, where the convexity of the vocal fold surface is noticeable and asymmetry of contacting surfaces may become apparent [14]. In vocal fold modeling, it is important to successfully detect contact at glottal exit, as collision forces acting in that specific area can be responsible for damages [4]. Furthermore, the choice of master faces and slave nodes cannot be made a priori due to the vocal 
fold geometry and a combinatorial search must be performed with the corresponding computational cost [17]. In vocal folds collision, the contact detection problem is therefore typically simplified by enforcing contact at the mid-saggital plane [10, 4, 9]. However, it is important to avoid this assumption, since asymmetries in the vocal fold dynamics play a role in abnormal phonation [28]. In the present work, a new vocal fold collision detection mechanisms is implemented within the Deformable Simplicial Complex (DSC) framework [29]. The DSC method is a topology-adaptive method for tracking deformable interfaces represented as a set of triangular faces separating a tetrahedral unstructured mesh in different mediums. The detection mechanism makes use of the airway mesh, as will be described next. It allows for asymmetric collision and it supports both the penalty and the Lagrange multiplier formulations. For the sake of simplicity, the tissue mesh topology is fixed during the deformation, and only the vertices are advected.

The proposed contact detection mechanism is based on oriented volume inversion of an intraglottal conforming tetrahedral mesh, connecting the two sides of the vocal folds, as shown in Figure 2. Once a conforming airway tetrahedron is inverted, the nodes $\mathbf{x}^{s}, \mathbf{x}_{k=1,2,3}$ of a nodeto-surface contact element can be defined, by making use of nodes labeling. As a consequence of the simplicity of the flow model, a criterion to stop the flow is defined. Hence, the flow is zero whenever any of the inverted airway tetrahedra has barycentric coordinates $\left(x_{b c}, y_{b c}, z_{b c}\right)$ such that $\left|y_{b c}\right| \leq y_{i} \cdot \tau$, where $\tau$ is a zero flux parameter and $y_{i}$ is the length of the major axis in Figure 1. By enforcing this condition, the Bernoulli airflow is assumed to be independent of variations in the anteroposterior dimension below a certain threshold defined by $\tau$.

The constrained contact problem can be solved by the Lagrange multiplier method in Eqs. (25). In order to avoid projection existence problems that may arise at glottal exit, the domain in which the algorithm seeks $\overline{\mathbf{x}}^{m}$ can be expanded. For a given relaxation parameter $\delta$ with area units, the slave node projects onto the master surface if

$$
\frac{1}{2}\left(\sum_{k=1,2,3} \sum_{l=1,2,3}\left\|\left(\mathbf{x}_{k}-\overline{\mathbf{x}}^{m}\right) \times\left(\mathbf{x}_{l}-\overline{\mathbf{x}}^{m}\right)\right\|_{2}-2 \cdot\left\|\left(\mathbf{x}_{2}-\mathbf{x}_{1}\right) \times\left(\mathbf{x}_{3}-\mathbf{x}_{1}\right)\right\|_{2}\right) \leq \delta,
$$

where $\|\cdot\|_{2}$ is the 2-norm distance. Since the condition is related to the geometry illustrated in Figure 2, the relaxation parameter is expected to depend on the tetrahedral mesh used for the computations. Therefore, a general value cannot be given, and only a trial and error approach can provide a good parameter.

As pointed out earlier, the penalty formulation in Eq. (22) can be simplified. Once a negative element volume $V^{e}$ is found, the compressive reaction force on a colliding element may be approximated numerically as

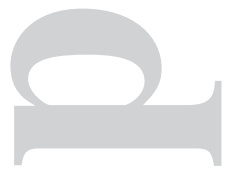

$$
-\left(\mathbf{g}^{e}\right)^{T} \kappa \mathbf{g}^{e} \cdot\left(\mathbf{x}^{s} \mathbf{x}_{1} \mathbf{x}_{2} \mathbf{x}_{3}\right)^{T} \approx(-\mathbf{n} \mathbf{n} \mathbf{n} \mathbf{n})^{T} \kappa \frac{V^{e}}{4}
$$

where $\mathbf{n}=\overline{\mathbf{n}}^{m}$ is the outward normal in Figure 2. Assembly may be performed to approximate the contact force vector $-\mathbf{G}^{T} \kappa \mathbf{G x}$ in Eq. (22), to be added to the external force vector $\mathbf{F}$. The algorithm safely avoids the classical master-slave paradigm, in which normal gap computations and projections are required. The contact algorithm is presented in Table I.

The collision approach is based in a coarse airway mesh that cannot present inner vertices. Therefore, an obvious drawback of the algorithm is the incompatibility with a numerical NavierStokes model of the flow.

\subsection{Numerical Solver}

Earlier in the paper, it was noted that the mechanical equilibrium of a system with positionbased constrains described by the Lagrange function occurs at a saddle point. This means that the system matrix in Eq. (25) is indefinite. Consequently, gradient methods, which are effective to solve symmetric positive definite linear systems of equations, cannot be employed in the present model. As described in [29], the iterative generalized minimum residual method [30] is used in this study.

To improve the performance of the method, the preconditioning technique based on [31,29] is well-suited for the present investigation. Hence, the system is preconditioned by multiplication of 
the inverse of a diagonal matrix

$$
\mathbf{P}=\left(\begin{array}{cc}
\operatorname{diag}(\mathbf{A}) & \mathbf{0} \\
& (1+\alpha) h^{2} \beta \cdot \operatorname{diag}\left(\left\{\mathbf{D}^{T} \mathbf{G}_{n+1}^{T}\right\}^{T} \cdot\right. \\
\mathbf{0} & \left.\cdot[\operatorname{diag}(\mathbf{A})]^{-1}\left\{\mathbf{D}^{T} \mathbf{G}_{n+1}^{T}\right\}\right)
\end{array}\right)
$$

at both sides of Eq. (25), where $\operatorname{diag}(\cdot)$ is a diagonal matrix with the diagonal elements in the argument, and $\{\cdot\}$ indicates an assembled matrix.

\section{SIMULATION SETUP}

The layered tissue geometry used in the simulations is adapted from [17], where elliptical shaped cross-sections are assumed, as stated earlier in Section 2.1; see Figure 1. The larynx is discretized into 51 cross-sections with constant major axis $y_{i}=0.7 \mathrm{~cm}$ and minor axis shown in Table II, and it is embedded into a cylinder of radius $0.8 \mathrm{~cm}$ and length $2 \mathrm{~cm}$. The geometrical description of the vocal folds correspond to a parallel and symmetric prephonatory profile, a glottal gap of 0.05 $\mathrm{cm}$, and vocal fold vertical thickness and lateral depth of $0.9 \mathrm{~cm}$ and $0.77 \mathrm{~cm}$, respectively. No false vocal folds are included. For an average height of the intraglottal interface triangles of $0.02 \mathrm{~cm}$, discretization of the narrow intraglottal airway in 9 sections (see $S_{8}$ to $S_{26}$ in Table II) is chosen to keep low resolution Bernoulli flow while self-sustained oscillations are assured.

The tissue properties can be found in Table III. The values $\rho=1.02 \mathrm{~g} / \mathrm{cm}^{3}$ and $\rho_{\text {air }}=1.23$. $10^{-3} \mathrm{~g} / \mathrm{cm}^{3}$ are chosen for the tissue and air density, respectively. The driving subglottal pressure is set to $p_{\text {sub }}=0.8 \cdot 10^{4} \mathrm{dyn} / \mathrm{cm}^{2}$. The initial tissue velocity is set to zero.

The value $\tau=0.5$ is chosen for the zero flow parameter, with the aim of not favoring extreme cases. The relaxation parameter for projection is set to $\delta=0.2$ by trial and error, leading to a condition in Eq. (27) that successfully detects inverted tetrahedral at glottal exit for the tetrahedral mesh used in the simulations. The default temporal discretization scheme for calculations is the implicit, second-order accurate Hilbert-Hughes-Taylor $\alpha$-method. The parameters employed are $\alpha=-0.3$ and time step increment $h=50 \mu \mathrm{s}$.

The finite element mesh consists of 24584, 12754 and 17646 tetrahedra for body, ligament and cover, respectively. With regard to the labeling, the tissue layers are labeled differently for the left $(x<0)$ and right $(x>0)$ vocal folds. Furthermore, linear shape functions are used for the spatial finite element discretization. The relative tolerance of the iterative generalized minimum residual algorithm is $10^{-8}$ in all simulations.

\section{RESULTS AND DISCUSSION}

\subsection{Model validation}

Validation of the present vocal fold model is done by comparison of typical outputs with published numerical results in [5, 17, 6, 4, 10, 32] and experimental results in [33, 34]. In this section, the contact model to be validated is solved by the Lagrange multiplier approach, and further validated by comparison with the commercial software ANSYS ${ }^{\circledR}$ Academic Research, Release 16.1.

The spatial coordinates of selected interface nodes placed on the mid-coronal plane are shown in Figure 3. The trajectories projected on a coronal plane are shown in Figure 3a, where circles indicate the initial configuration, making the initial transient apparent. The gray dashed line illustrates the initial superficial state of the vocal fold. The amplitude of oscillation in the vertical direction increases as the nodes approach the glottal exit, in agreement with the results in [17]. However, notice that the trajectories slightly differ from the paths measured on excised larynges [34]. In the present collision study, the choice of the vocal fold tissue parameters is made to simulate significant vocal fold contact to the detriment of highly realistic trajectories. Furthermore, Figure $3 \mathrm{~b}$ shows the waveforms of the $x$ spatial coordinates of nodes 6157 and 6390 in Figure 3 a with initial material coordinates $(0.0250,0,-0.1077)$ and $(0.0500,0,-0.3519)$, respectively. The oscillations in the first $0.02 \mathrm{~s}$ are omitted for clarity reasons. The nodes present admissible theoretical amplitudes of oscillation [5]. The fundamental frequency of oscillation is about $219 \mathrm{~Hz}$, which is an acceptable value for female phonation voices. Node 6157 placed in the narrow glottal region is affected by 
collision, which is apparent from the waveform near the mid-saggital plane $(x=0)$. Compared to the results in [17], in the present model collision forces act to avoid body interpenetration. Figure 4 shows a coronal view of the glottal shape during a glottal cycle. The model has the ability to capture the alternate convergent-divergent shapes $[35,36]$. A diverging shape can be observed during the closing phase (Figure 4a), followed by a converging one at collision (Figure 4b), which is maintained throughout the opening phase (Figures $4 c-d$ ). Notice in Figure $4 b$ the significant contact region.

In Figure 5 the volumetric flow rate during transient and steady oscillations is shown. The maximum flow is between 500 and $600 \mathrm{~cm}^{3} / \mathrm{s}$. For a Bernoulli flow and similiar initial cross-sectional areas [6], this is a feasible value. Furthermore, the open quotient in the simulations is about 0.87 , slightly lower than the value reported in the computational model of [5]. Nevertheless, this value is above typical experimental data reported in the literature [32]. High open quotients have been shown to be related to high fundamental frequencies [32], and low subglottal pressures [17]. In the present model, a decrease of the subglottal pressure from $0.8 \mathrm{kPa}$ to $0.6 \mathrm{kPa}$ produces an increase of the open quotient from 0.87 to 0.89 , and a frequency increase from $219 \mathrm{~Hz}$ to $222 \mathrm{~Hz}$, in agreement with the results in [17]. Hence, with regard to the closing time, the present model is capable to simulate a similar trend as observed in previous studies. Finally, the open quotient highly depends on the solution method, as will be shown in the following section.

The performance of the proposed collision method is firstly examined by comparison to numerical solutions obtained with the commercial software ANSYS ${ }^{\circledR}$. A simple impact problem is defined as two solid cylinders with the same dimensions and initial velocities $35 \mathrm{~m} / \mathrm{s}$, placed at a distance of $0.007 \mathrm{~m}$; see Figure 6a. The material of the two cylinders is considered linear elastic with complete isotropy. The Young's modulus is $E=2 \cdot 10^{9}$ and the Poisson's ratio $\nu=0.3$. Frictionless contact is assumed. For comparison reasons, the contact problem in the commercial software is formulated in a Lagrangian form, and the contact searching algorithm is based on a master-slave configuration (target-contact in ANSYS terminology) with normal direction from the master surface, which corresponds to the cylinder at the left of Figure 6a. However, given the symmetry of the problem, the auto-symmetric behavior option in ANSYS ${ }^{\circledR}$ is activated. This means that the slave and master surface can be reversed locally. In the proposed collision model, the coarse conforming mesh for contact detection is generated in a region of possible contact defined a priori, in this case between the two cylinders in the cube $[0,2.7] \times[-1,1] \times[-1,1]$. Notice that some boundary elements of the conforming mesh are partially visible in Figure $6 \mathrm{~b}$ to illustrate the region of possible contact. Figure $6 \mathrm{~b}$ shows the deformation of the colliding cylinders at times $t=0.0011 \mathrm{~s}$ (top) and $t=0.003 \mathrm{~s}$ (bottom) obtained with the proposed contact model (left) and ANSYS ${ }^{\circledR}$ (right). Only the boundary triangles are shown in the custom simulations for clarity reasons. The deformations are reasonable and show good agreement between the two simulations. Furthermore, the contact searching algorithm based on tetrahedral volume inversion seems to successfully detect contact. The selected times correspond to peak values of the total contact force, as Figure 7 shows. In Figure $7 \mathrm{a}$ the magnitude of the total contact force applied to each of the cylinders is shown. The similarity between the two curves seems to be in agreement with the action-reaction principle. In Figure $7 \mathrm{~b}$ the total contact force applied to one of the cylinders is compared to the results obtained with ANSYS ${ }^{\circledR}$. The difference between the two simulations is between $2.8 \%$ and $6 \%$ for extreme values. This deviation may be explained by the relaxation of the projection condition in Eq. (27), which can lead to slight overestimation of the reaction forces. Furthermore, the results obtained with the proposed collision methodology show a compressive stress peak of about $4.89 \cdot 10^{5} \mathrm{kPa}$, which is $1.2 \%$ lower than the maximum compressive stress $4.95 \cdot 10^{5} \mathrm{kPa}$ computed in ANSYS. Therefore, the proposed contact model provides numerical solutions similar to the results obtained with the cited commercial software for simple impact problems.

In regard to vocal fold collision, Figure 8 shows a saggital view of the vocal folds and the magnitude of the $x$ component of the contact and aerodynamic forces acting on the right vocal fold $(x>0)$ during vocal fold separation, at contact times $t=0.0371,0.0372 \mathrm{~s}$, from top to bottom. The contact force at each of the interface triangle is computed from the summation of contact forces applied to the vertices. Above the contact region the force is zero, since no vocal tract coupling is considered in this model. From the figure, the contact detection mechanism seems to successfully detect contact at glottal exit. In agreement with the studies presented in [10], maximum contact force occurs at the mid-coronal plane. With regard to the pressure distribution in the anteroposterior direction, for a minimum prephonatory glottal half widths of $0.025 \mathrm{~cm}$ between $z=-0.25$ and $-0.03 \mathrm{~cm}$, collision occurs along approximately $40 \%$ of the whole length of the vocal fold, which is similar to the results obtained in [10]. As in [4], values are of order of magnitude $10^{-4}$ in $\mathrm{N}$. 
Finally, the relation between peak contact stress values and subglottal pressures is demonstrated in Table IV. The mediolateral component of the contact stress appears to increase as a function of the suglottal pressure, in agreement with the normal stress measurements in [33]. A simple linear regression reveals a slope of 1.72 and a coefficient of determination $\mathrm{R}^{2}$ of 0.989 . Furthermore, the estimated values belong to the interval $[0.41 .5] \mathrm{kPa}$. All these values are similar to the ones reported in [33].

\section{r. \\ 4.2. Contact constraint enforcement}

The vocal fold contact problem is here solved by the Lagrange multiplier method, and a penalty method with the approximation in Eq. (28), which takes advantage of the conforming airway mesh. The penalty method is applied to investigate the effect of inexact position-based contact constraint enforcement [9] and spring-like contact forces on the dynamics and mechanics at vocal fold contact. These results are compared to the proposed Lagrange multiplier solution for vocal fold contact.

Figure 9 shows the results obtained with the Lagrange multiplier method (solid line), and the results obtained with a penalty method with $\kappa=10^{7}$ (dotted line). In Figure 9a waveforms of the $x$ spatial coordinate of two opposite interface nodes placed at initial material coordinates $(0.024,-0.136,-0.037)$ and $(-0.024,-0.136,-0.037)$ are shown. The figure illustrates how the Lagrange multiplier solution is capable to simulate realistic displacements, while the penalty solution exhibits a noticeable volume interpenetration. In Figures $9 \mathrm{~b}$ and $9 \mathrm{c}$ the $x$ component of the tissue velocity and the nodal contact force applied to an interface node with initial position $(0.024,-0.136,-0.037)$ is shown, respectively. It is noted a spurious non-smooth behavior that arises at collision in the Lagrange multiplier solution. It may be argued that the abrupt changes in nodal contact force calculation are a consequence of the non-smoothness introduced by the contact conditions in Eq. (11). Regularization of the exact impact solution is common practice in numerical mechanics, which can be performed by several techniques. For instance, in [37] the Lagrange multipliers are computed at a time prior to collision instead, with a contact matrix built from the collision positions. Physically, the resulting smoothing effect may be explained by the compressibility of the air previous to collision modeled by the Lagrange multipliers. Nevertheless, an explicit scheme has to be used to temporally discretize the problem, as explained in [37]. These schemes are not unconditionally stable, which is not a desirable feature for complex mechanical problems as the present one. Alternatively, the solution may be regularized by means of other methods such as the Augmented Lagrange method [25], or Nitsche's method [38]. However, notice in Figure $9 \mathrm{c}$ that temporal integration of the two solutions give similar results. This observation suggests that the spatially integrated Lagrange contact force may show a different trend. This is supported by the results in Figure 10 that shows the mediolateral component of the impact stress computed with the Lagrange multipliers and the penalty methods, for a subglottal pressure of 0.8 $\mathrm{kPa}$. The strong non-smooth behavior of the nodal contact force in Figure 9c is less apparent. In contrast to the penalty solution, the resulting Lagrange stress curve presents a sudden increase at first moment of impact, followed by a quick decrease. Then, the pressure decreases gradually, with a small positive increment which precedes the final contact phase. This behavior is in very good agreement with the impact pressure measurement results in [33], that show a sharp pressure pulse at the impact phase, and a pressure buildup in the preopen phase. This final impact pressure increase is also apparent in the results for the cylinders problem in Figure 7 . The penalty solution, however, fails at reproducing the peak stress consequence of the high kinetic energy previous to collision; notice the high tissue velocities previous to impact in Figure 11b. This reveals the limitation of linear spring-like vocal fold contact forces to quickly dissipate the large kinetic energy at impact. Previous studies on collision forces and tissue stresses [4] have shown that large collision forces may be a cause of tissue damage. Hence, a penalty vocal fold contact model may lack relevant information related to damage, and discrete vocal fold models may consider the use of non-linear spring models of contact.

The impact stress in Figure 10 show a large vertical gap between the two curves. Comparison with the results in Figure 9c makes apparent that contact area calculations are significantly affected by the solution method. Furthermore, as mentioned earlier in this paper, accurate contact dynamics estimation, such as contact area, is of importance in inverse problem formulations [8]. In addition, contact areas and collision forces have been shown to be dependent on uncertain parameters, such as the subglottal pressure [4]. Therefore, robust and good estimates of the dynamical behavior at 
contact are necessary for voice pathology analysis. The numerical study that follows investigates the performance of different theoretical models of the vocal folds with regards to features estimation.

The importance of enforcement of the position-based collision constraint is illustrated in Figure 11. In Figure 11a the maximum contact area computed from the summation of triangular interface areas with vertex loaded by a collision force is shown; the horizontal axis corresponds to the interpenetration volume normalized to the maximum intersection volume when the effect of contact forces is neglected. Furthermore, the results are shown for a subglottal pressure of $p_{\text {sub }}=0.8 \mathrm{kPa}$ in black marks, and $p_{\text {sub }}=0.6 \mathrm{kPa}$ in white marks. The general increasing trend of the values suggests that overestimation of the contact area may occur as the non-penetration condition relaxes. In the same figure, circles correspond to the results of the Lagrange multiplier method; downward triangles, squares, and upward triangles correspond to the penalty formulation with $\kappa=10^{7}, 10^{6}$ and $10^{5}$, respectively. The maximum value of the penalty parameter shown in the results is constrained to the ill-conditioning of the system matrix and physical meaning of contact forces. The results show that the penalty parameter has a direct influence on contact area prediction. Furthermore, open quotients values of $0.85,0.76$ and 0.7 are predicted for the reported penalty parameters, respectively, with a subglottal pressure of $p_{s u b}=0.8 \mathrm{kPa}$. This means that inexact position-based contact constraint enforcement has an effect on the temporal distribution of the transfer of kinetic energy at impact. Furthermore, in [17] it is argued that high kinetic energy is related to low open quotients. Inspection of Figure 11a reveals slight larger amplitudes of the penalty solution compared to the Lagrange multipliers solution, which gives an open quotient of 0.87 . Hence, increasing interpenetration leads to a spurious increase of the kinetic energy and contact areas, and decreasing open quotients, in agreement with [17]. Finally, note that small subglottal variations do not have a strong effect on contact area estimations, as large contact area variations in the penalty solution may be explained by spurious kinetic energy variations. Therefore, the contact area is dependent to subglottal pressures, as predicted in [4], but also highly dependent to the contact constraint enforcement.

In regard to contact force estimation, Figure $11 \mathrm{~b}$ shows the maximum $x$ component of the total contact force. The observed general trend indicates an effect of the vocal fold interpenetration, as expected from Eq. (28); hence, the value of the penalty parameter is crucial. Furthermore, comparison of collision forces with $p_{s u b}=0.8 \mathrm{kPa}$ (black marks) and $p_{s u b}=0.6 \mathrm{kPa}$ (white marks), shows that contact forces decrease with subglottal pressure, regardless of the method used to enforce the contact constraint. Finally, note that the peak impact stress in Figure 10 does not correspond to the ratio between maximum total contact force and maximum contact area in Figure 11 as peak values do not occur at the same time, in agreement with [4].

Due to the difficulties in extracting experimental model parameters, arbitrary choice of the input contact parameters is generally made [6]. Hence, the number of unknown input parameters introduces uncertainty in the model output, as demonstrated earlier. In this sense, the Lagrange multiplier method appears to be a solution to reduce model uncertainties by reducing the number of unknowns and introducing robustness. Finally, notice that the solution obtained by the Lagrange multiplier method reduces the non-physical interpenetration volume by a factor of $10^{3}$, but still it is not zero as one may expect from the theory. This can be explained as a consequence of numerical errors and the relaxed definition of slave node projection in Eq. (27).

In summary, inexact contact constraint enforcement has a clear effect on vocal fold dynamics. The proposed Lagrange vocal fold contact solution is capable to successfully replicate the temporal distribution of the impact stress in contrast to linear spring-like contact forces. Furthermore, it is able to introduce robustness and reduce uncertainties in the model output. Nevertheless, the Lagrange approach may lead to under and overestimation of the instantaneous nodal contact force and the tissue velocity at contact.

\subsection{Temporal integration}

Earlier in Section 2.3.2, the importance of temporal integration schemes with respect to system dynamics was emphasized. A numerical study of a number of schemes that are unconditionally stable and second-order accurate under certain of premises (see Section 2.3.2) is here presented. Due to the complexity of the system, methods with stability conditions such as the $\alpha$-method with $\alpha=-0.41421$ used in [39] are not considered.

In regard to nodal force collision calculations, the temporal integration scheme appears to have an influence as Figure 12 shows. In the figure, circles correspond to the undamped Newmark scheme in Eq. (24) with $\gamma=\frac{1}{2}$ and $\beta=\frac{1}{4}$, similar to e.g., [9]; triangles indicate the $\alpha$-method with $\alpha=-0.3$; 
solid and dotted lines indicate the results with the Lagrange multiplier and the penalty methods $\left(\kappa=10^{7}\right)$, respectively. The spurious non-smooth behavior worsens for an undamped Newmark method. It might be argued that the lack of negative numerical damping leads to an increase of inertial forces and large amplitude of oscillation, and thereby a worsening of the non-smoothness. As expected, large amplitudes of vocal fold oscillation have been observed with the undamped temporal scheme, although the results are not shown here for the sake of brevity. Notice that the increase of amplitude has a clear a effect on the estimation of the penalty nodal contact force solution, in agreement with the results in Figure 11. Furthermore, no noticeable phase error can be seen from the temporal discretization simulations. Finally, the numerical solutions have a stable behavior with a time step of $50 \mu$ s, which supports the unconditional stability condition.

In summary, the results suggest that $\alpha$-methods are superior to Newmark schemes as numerical damping appears to be advantageous in vocal fold contact simulations.

\section{(

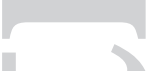 \\ 4.4. Asymmetric collision study}

Asymmetric vocal fold oscillations are typically observed in several voice pathologies such as mass lesions, intracordal scarring or vocal fold paralysis [28]. This has motivated numerical studies on the effect of abnormal oscillations on phonation $[12,15]$. In contrast to previous studies on collisionfree asymmetric oscillations [12], the proposed contact detection algorithm allows for the study of the effect of asymmetric collision on vocal fold mechanics. Similarly to [12, 15], the present work limits to asymmetric vibration induced by a tension imbalance between left and right cover layer. Thus, structural asymmetries typical of mass lesions are not included, which would possibly require the modeling of frictional contact. All simulations use a constant subglottal pressure of $0.8 \mathrm{kPa}$.

A tension mismatch $100 \cdot\left(E_{\text {right }}-E_{\text {left }}\right) / E_{\text {right }}$ of $20 \%$ and $40 \%$ between left and right longitudinal Young's modulus of the cover layer is enforced. This means that the right vocal fold is stiffer than the left vocal fold. Figure 13 compares the left and right vocal fold mechanical response averaged over 4 oscillatory cycles immediately after the initial transient response (see Figure 5). The results with symmetric mechanical properties are shown for comparison reasons. All values correspond to the arithmetic mean. To illustrate the asymmetric oscillations, solid horizontal lines indicate the $x$ coordinate of the collision point of node 6157 , normalized to the $x$ coordinate of its initial material coordinate for clarity reasons. From the results, the collision point appears to be successfully detected by the contact detection algorithm. Furthermore, black dot clips correspond to the ratio between peak-to-peak $x$ displacement of node 6157 and the opposite node laying on the left vocal fold; this is equivalent to a classical measure of asymmetry in clinical research [28]. In the figure, the ratio seems to increase with the tension mismatch, indicating increasing asymmetries in the amplitude of vibration. In dashed lines, the collision-free solution is shown by neglecting the effect of surface contact forces. These results indicate that the collision forces, and thereby the energy distribution, play a role on asymmetric vocal fold self-oscillations. In connection with the results in Figure $11 \mathrm{~b}$, inexact contact constraint enforcement, widely accepted in the vocal fold modeling community, has an effect on asymmetric dynamics.

With regard to internal and reaction forces, gray and white bars in Figure 13 show the maximum compressive stress and the $x$ component of the Lagrange solution of the contact force integrated over a collision interval, respectively. As the stiffness asymmetry increases, an increasing compressive stress is apparent. It can be hypothesized that asymmetric oscillations increase the risk of damage since large compressive stress may be related to vocal fold damage [3]. Nevertheless, impact forces do not appear to be correlated with the degree of asymmetry. Previous studies have related mass lesions with impact forces at vocal fold collision [40]. Hence, from the present results asymmetric collision can not be considered a sufficient factor for increasing risk of damage.

Furthermore, note in Figure 13 that the same mediolateral total contact force is applied to each vocal fold, and similarly, the small anteroposterior component, which is of order $10^{-6} \mathrm{~N}$. This contradicts a number of results in [15], where asymmetry in the vocal fold oscillation is related to asymmetry in the anteroposterior component of the contact force. However, the action-reaction principle predicts equal forces, and hence, the present simulations validate the contact model proposed in this work. Finally, it is noteworthy that variances increase with tension imbalance, which may indicate unstable oscillation, as investigated in [6]. 


\section{CONCLUSIONS}

The asymmetric collision problem of a three-dimensional continuum model of the vocal folds driven by Bernoulli aerodynamic forces has been formulated in terms of position-based inequality constraints. In contrast to existing symmetric vocal fold contact models [17, 10, 15], the present contact model has been developed within a finite-element contact mechanics framework, providing mathematical details and a numerical analysis. This work covered the entire contact modeling process, which includes definition of contact boundary condition, contact detection methodology, validation and numerical analysis.

A novel contact detection mechanism has been developed to solve the problems of symmetric contacting bodies, non-smooth boundaries, and asymmetric contact detection posed in [9] for the general case of non-matching tissue meshes. Firstly, the algorithm safely eliminates the need of predefinition of the master and the slave bodies, which poses a problem for vocal folds with a symmetric geometry. Secondly, it deals with the projection point problem at convex boundaries such as the upper vocal fold surfaces. Hence, the algorithm successfully detects contact at glottal exit where collision forces may be responsible for tissue damage [4]. Thirdly, the models successfully detects asymmetric contact induced by tissue property asymmetries. However, the model may show limitations in voice pathologies investigations related to geometrical irregularities as frictional contact is expected to play a role. Nevertheless, the proposed node-to-surface contact model with the novel contact detection mechanism is extensible to frictional contact with relative ease. Therefore, future developments of the model will be devoted to vocal fold contact in asymmetric geometries.

Numerical analysis has been included in this study to assure a good performance of the vocal fold model. The effect of inexact collision constraint enforcement $[9,15,11]$ and temporal integration schemes on vocal fold mechanics and dynamics has been investigated, the results showing that vocal fold contact is highly sensitive to non-physical energy variations. Different variational methodologies for constrained minimization problems have been applied to the vocal fold contact problem. It has been shown that linear spring-based contact models poorly replicate the time evolution impact phase observed in vocal fold measurements [33]. Furthermore, the penalty solution is highly sensitive to subglottal pressure variations and model parameter-choice. In an effort to reduce uncertainties in the model output and spurious energy variations, a vocal fold contact model based on the Lagrange multiplier method has been proposed. To reduce the computational cost of the Lagrange solution, a preconditioner to increase the convergence ratio of the iterative solver has been designed. The solution showed realistic contact stress simulations, robustness in terms of subglottal pressure variations, and accuracy in vocal fold contact dynamics estimation. However, the method is not suitable for predicting instantaneous nodal contact forces, which showed a spurious non-smooth behavior. Finally, the non-smooth vocal fold collision problem benefits from highly dissipative temporal discretization schemes. A Hilber-Hughes-Taylor $\alpha$-method with $\alpha=-0.3$ seems to provide satisfactory results.

The present contact model is capable to predict specific mechanical behavior at asymmetric contact induced by stiffness mismatch enforcement, in contrast to the collision-free study in [12]. In contrast to the trend observed in impact force estimations, an increasing maximum compressive stress seems to correlate with the degree of asymmetry, which may contribute to additional damage.

Finally, the present finite-element vocal fold contact model may be adapted to advanced fluidstructure models with simplified symmetric contact (e.g., see [9, 11, 2]) to provide more insights into the effect of collision on phonation.

\section{ACKNOWLEDGEMENT}

This work was supported by grant number 110230 from the Swedish organization AFA Försäkring.

\section{REFERENCES}

1. Van Den Berg J, Zantema JT, Doornenbal RJ. On the Air Resistance and the Bernoulli Effect of the Human Larynx. J. Acoust. Soc. Am. 1957; 29(5):626-631,

2. Šidlof P, Zörner S, Hüppe A. A hybrid approach to the computational aeroacoustics of human voice production. Biomech. Model Mechanobiol. 2015; 14(3):473-488.

3. Titze IR, Svec JG, Popolo PS. Vocal dose measures: quantifying accumulated vibration exposure in vocal fold tissues. J. Speech Lang. Hear. R. 2003; 46(4):919-932. 
4. Gunter HE. A mechanical model of vocal-fold collision with high spatial and temporal resolution. J. Acoust. Soc. Am. 2003; 113(2):994-1000.

5. Alipour F, Berry DA, Titze IR. A finite-element model of vocal-fold vibration. J. Acoust. Soc. Am. 2000; 108(6):3003-3012.

6. Steinecke I, Herzel H. Bifurcations in an asymmetric vocal-fold model. J. Acoust. Soc. Am. 1995; 97(3):1874-84.

7. Horaček J, Sidlof P, Švec JG. Numerical simulation of self-oscillations of human vocal folds with Hertz model of impact forces. J. Fluid. Struct. 2005; 20(6):853-869,

8. Spencer M, Siegmund T, Mongeau L. Determination of superior surface strains and stresses, and vocal fold contact pressure in a synthetic larynx model using digital image correlation. J. Acoust. Soc. Am. Feb 2008; 123(2):1089_ 1103.

9. Zheng X, Xue Q, Mittal R, Beilamowicz S. A coupled sharp-interface immersed boundary-finite-element method for flow-structure interaction with application to human phonation. J. Biomech. Eng. 2010; 132(11):111 003.

10. Tao C, Jiang JJ, Zhang Y. Simulation of vocal fold impact pressures with a self-oscillating finite-element model. $J$. Acoust. Soc. Am. 2006; 119(6):3987-3994.

11. Bhattacharya P, Siegmund TH. Computational modeling of vibration-induced systemic hydration of vocal folds over a range of phonation conditions. Int. J. Numer. Meth. Biomed. Engng. 2014; 48:169-187.

12. Zhang Z, Hieu Luu T. Asymmetric vibration in a two-layer vocal fold model with left-right stiffness asymmetry: Experiment and simulation. J. Acoust. Soc. Am. 2012; 132(3):1626.

13. Xue Q, Mittal R, Zheng X, Bielamowicz S. A computational study of the effect of vocal-fold asymmetry on phonation. J. Acoust. Soc. Am. 2010; 128(2):818-827.

14. Simo J, Laursen T. An augmented lagrangian treatment of contact problems involving friction. Comput. Struct. 1992; 42(1):97-116, .

15. Xue Q, Mittal R, Zheng X, Bielamowicz S. Computational modeling of phonatory dynamics in a tubular threedimensional model of the human larynx. J. Acoust. Soc. Am. 2012; 132(3):1602.

16. Wriggers P. Computational contact mechanics. John Wiley \& Sons, 2002.

17. Rosa MDO, Pereira JC, Grellet M, Alwan A. A contribution to simulating a three-dimensional larynx model using the finite element method. J. Acoust. Soc. Am. 2003; 114(5):2893-2905.

18. Sommer DE, Erath BD, Zanartu M, Peterson SD. Corrected contact dynamics for the Steinecke and Herzel asymmetric two-mass model of the vocal folds. J. Acoust. Soc. Am. 2012; 132(4):EL271.

19. Story BH, Titze IR. Voice simulation with a body-cover model of the vocal folds. J. Acoust. Soc. Am. 1995; 97(2):1249-1260

20. Ishizaka K, Matsudaira K. Fluid mechanical considerations of vocal cord vibration. Monogr. 8, Speech Commun. Res. Lab.: Santa Barbara, CA., 1972.

21. Pelorson X, Hirschberg A, Van Hassel RR, Wijnands APJ, Auregan Y. Theoretical and experimental study of quasisteady-flow separation within the glottis during phonation. Application to a modified two-mass model. $J$. Acoust. Soc. Am. 1994; 96(6):3416-3431.

22. Berry DA, Titze IR. Normal modes in a continuum model of vocal fold tissues. J. Acoust. Soc. Am. 1996; 100(5):3345-3354.

23. Hughes TJR. The Finite Element Method: Linear Static and Dynamic Finite Element Analysis. Dover, 2000.

24. Zienkiewicz O. The finite element method. McGraw-Hill, 1977.

25. Bertsekas D. Constrained optimization and Lagrange multiplier methods. Athena Scientific, 1982.

26. Newmark NM. A Method of Computation for Structural Dynamics. J. Eng. Mech. Div. 1959; 85:67-94.

27. Hilber HM, Hughes TJR, Taylor RL. Improved Numerical Dissipation for time Integration Algorithms in Structural Dynamics. Earthquake Eng. Sruct. Dyn. 1977; 5:283-292, .

28. Bless D, Hirano M, Feder RJ. Videostroboscopic evaluation of the larynx. Ear, Nose \& Throat Journal 1987; 66:289296.

29. Misztal MK, Erleben K, Bargteil A, Fursund J, Christensen BB, Andreas Bærentzen J, Bridson R. Multiphase flow of immiscible fluids on unstructured moving meshes. IEEE Trans. Vis. Comput. Graphics 2014; 20(1):4-16,

30. Saad Y, Schultz MH. GMRES: A Generalized Minimal Residual Algorithm for Solving Nonsymmetric Linear Systems. SIAM J. Sci. Comput. 1986; 7(3):856-869.

31. Phoon KK, Toh KC, Chan SH, Lee FH. An efficient diagonal preconditioner for finite element solution of Biot's consolidation equations. Int. J. Numer. Meth. Eng. 2002; 55(4):377-400.

32. Lohscheller J, Švec J, Döllinger M. Vocal fold vibration amplitude, open quotient, speed quotient and their variability along glottal length: kymographic data from normal subjects. Logoped. Phoniatr. Vocol. 2013; 38(4):18292.

33. Jiang JJ, Titze IR. Measurement of vocal fold intraglottal pressure and impact stress. J. Voice 1994; 8(2):132-144.

34. Döllinger M, Berry DA. Visualization and quantification of the medial surface dynamics of an excised human vocal fold during phonation. J. Voice 2006; 20(3):401-413.

35. Titze IR. The Myoelastic Aerodynamic Theory of Phonation. National Center for Voice and Speech, 2006.

36. Zheng X, Mittal R, Xue Q, Bielamowicz S. Direct-numerical simulation of the glottal jet and vocal-fold dynamics in a three-dimensional laryngeal model. J. Acoust. Soc. Am. 2011; 130(1):404-415.

37. Carpenter NJ, Taylor RL, Katona MG. Lagrange constraints for transient finite element surface contact. Int. J. Numer. Meth. Eng. 1991; 32(1):103-128, .

38. Nitsche J. Über ein Variationsprinzip zur Lösung von Dirichlet-Problemen bei Verwendung von Teilräumen, die keinen Randbedingungen unterworfen sind. Abh. Math. Semin. Univ. Hambg. 1970; 36(1):9-15,

39. Bhattacharya P, Siegmund T. A computational study of systemic hydration in vocal fold collision. Comput. Methods Biomech. Biomed. Engin. 2013; 17(16):1835-52.

40. Titze IR. Mechanical stress in phonation. J. Voice 1994; 8(2):99-105, 


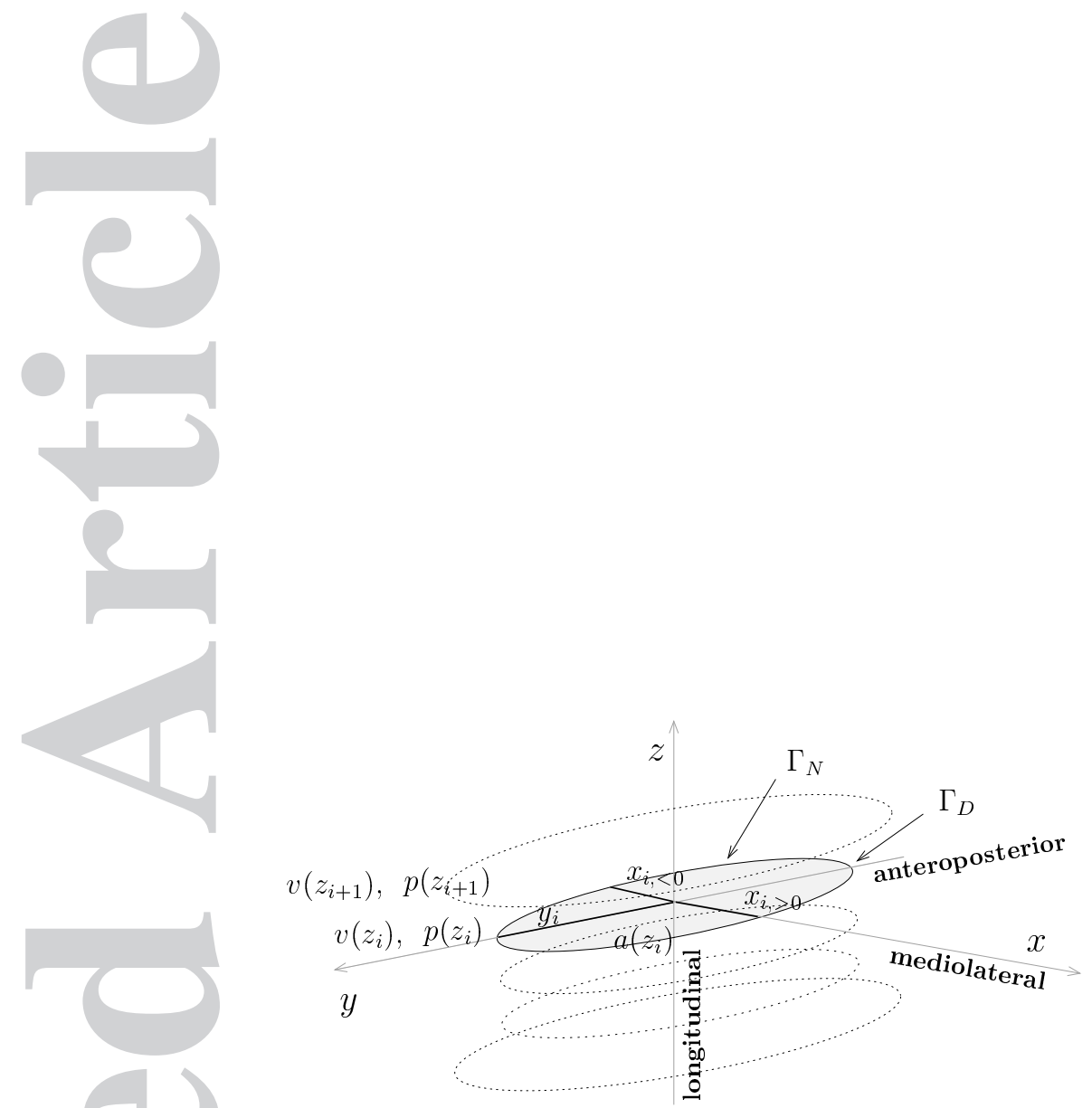

Figure 1. Schematic representation of the larynx assuming elliptical shaped cross-sections. Subscripts indicate spatial discretization along the longitudinal axis. $p\left(z_{i}\right), v\left(z_{i}\right)$ and $a\left(z_{i}\right)$ are the aerodynamic pressure, average flow velocity and cross-sectional area. $y_{i}$ is the major axis in the anteroposterior direction, and $x_{i,>0}$ and $x_{i,<0}$ are the positive and negative minor axes in the mediolateral direction, respectively. $\Gamma_{N}$ and $\Gamma_{D}$ indicate the Neumann and Dirichlet boundary, respectively. 

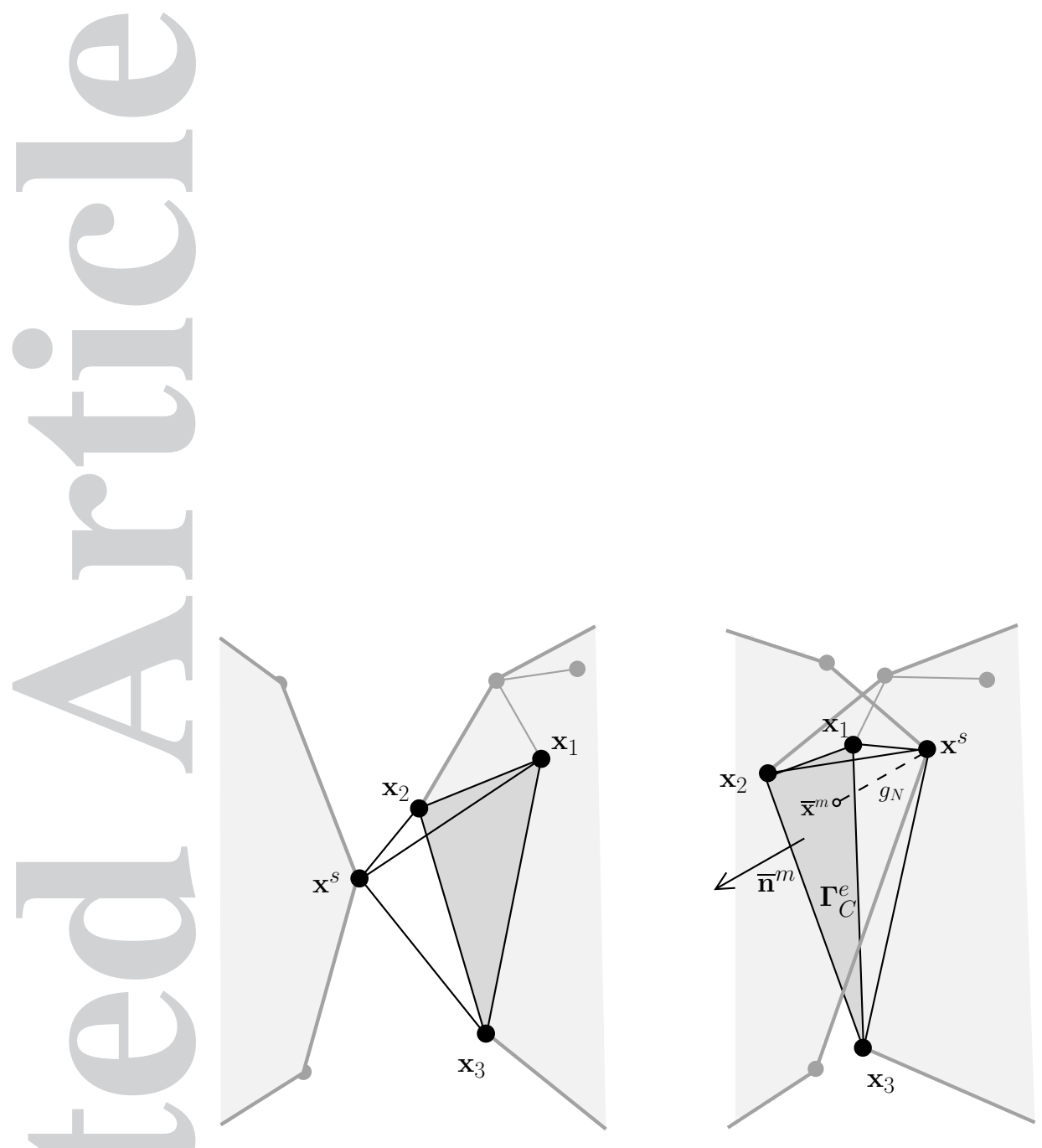

Figure 2. Schematic representation of the conforming interface mesh for collision detection; transverse view before collision (left), after collision (right). $\Gamma_{C}^{e}$ indicate the surface boundary of the node-to-surface contact element. 


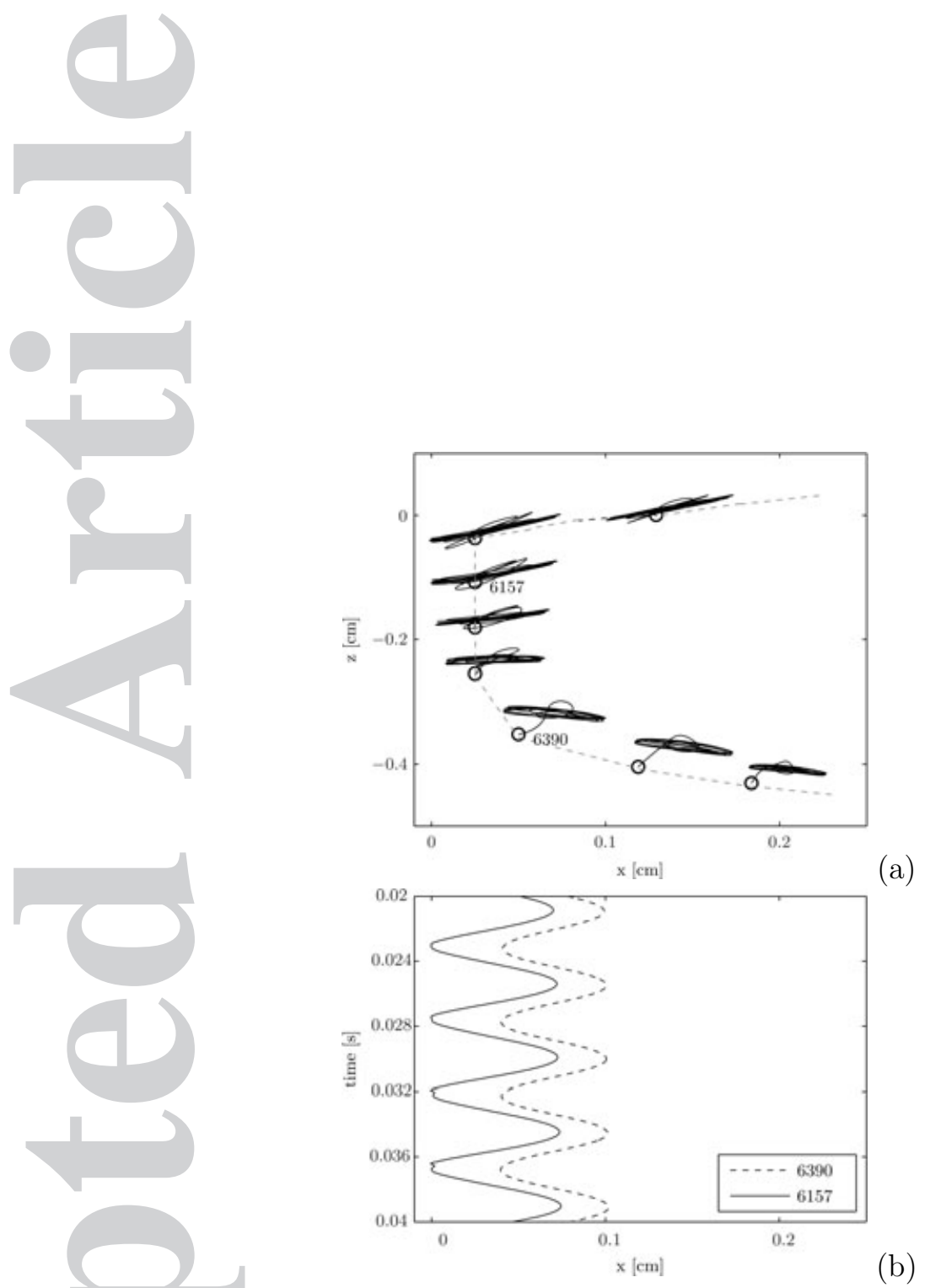

Figure 3. (a) Projected trajectories of selected interface nodes on the mid-coronal plane during the first 0.04 s. Circles corresponds to the initial configuration. Labels of two nodes, 6157 and 6390, with initial material coordinates $(0.0250,0,-0.1077)$ and $(0.0500,0,-0.3519)$, respectively, are shown. (b) Waveform of $x$ coordinate (mediolateral direction). Solid line corresponds to node 6157 , and dashed line to node 6390 . 


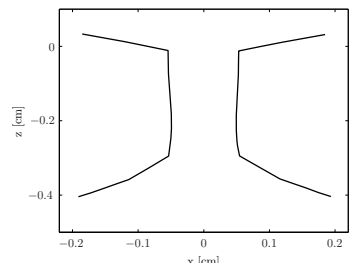

(a)

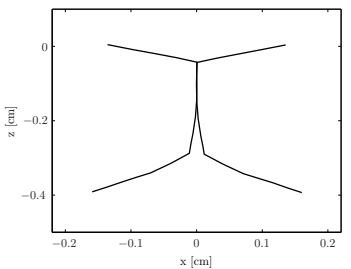

(b)

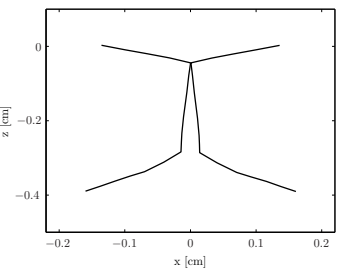

(c)

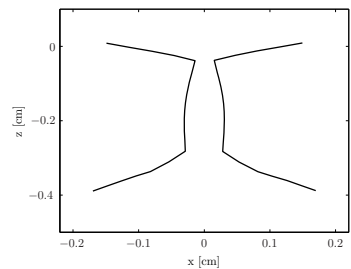

(d)

Figure 4. Vibration pattern for a subglottal pressure $0.8 \mathrm{kPa}$ at times $t=0.0353,0.0366,0.0367,0.0375 \mathrm{~s}$ from left to right, respectively.
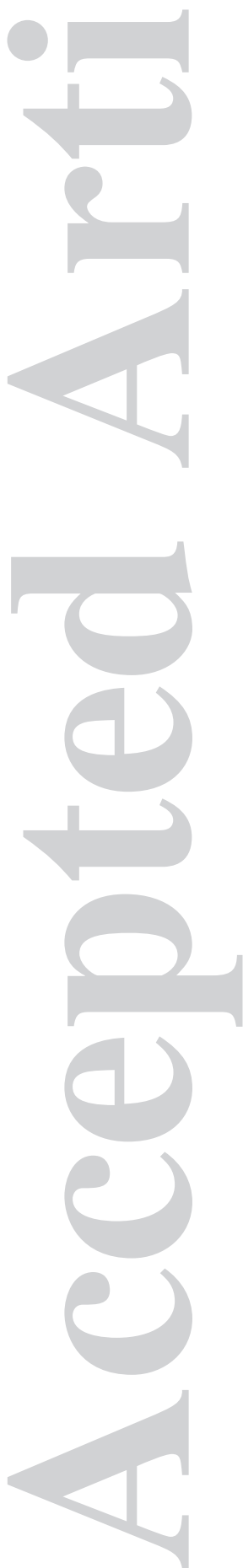

This article is protected by copyright. All rights reserved. 

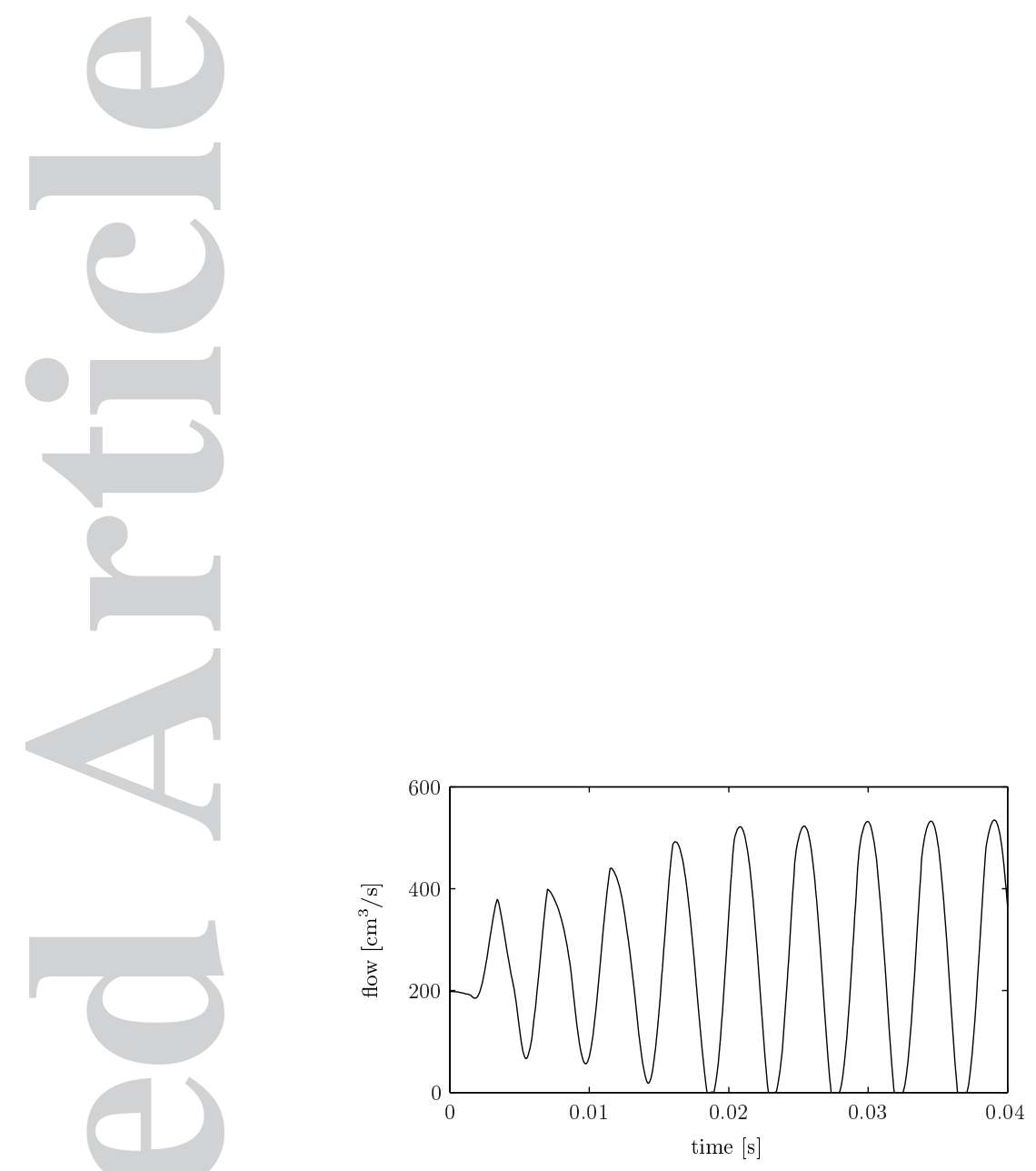

Figure 5. Volumetric flow rate $U$ approximated as in Eq. (2). Approximate steady oscillatory behavior follows an initial transient of duration about $0.02 \mathrm{~s}$. 


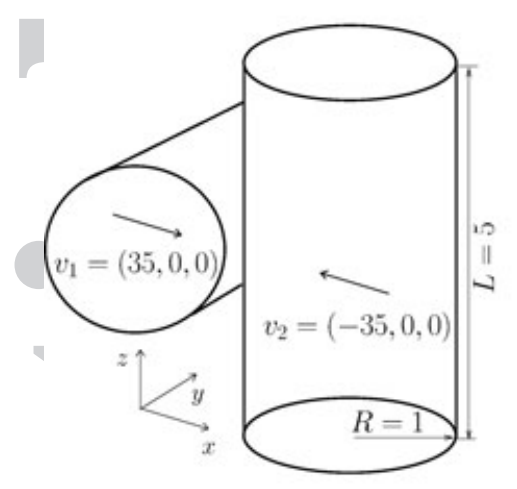

(a)
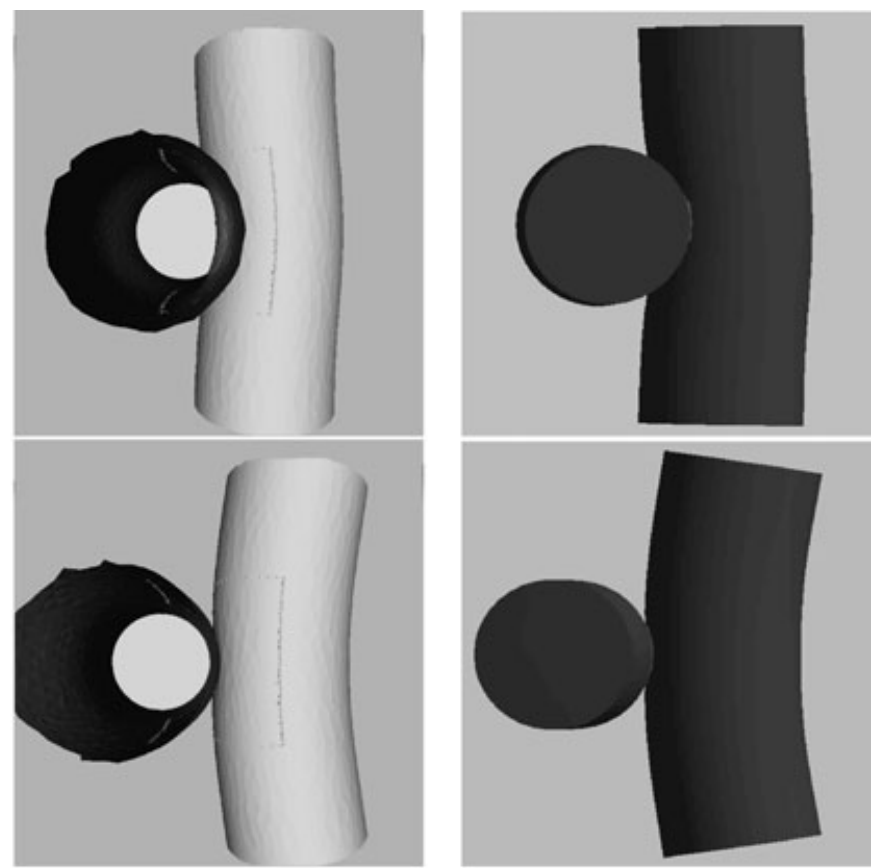

(b)

Figure 6. (a) Geometry of the impact problem. The origin is located at the center of the cylinder at the left of the illustration. All values in the SI of units. (b) Simulation of two colliding cylinders using the custom collision mechanism (left) and the commercial software ANSYS (right) at times $t=0.0011 \mathrm{~s}$ (top) and $t=0.003 \mathrm{~s}$ (bottom).

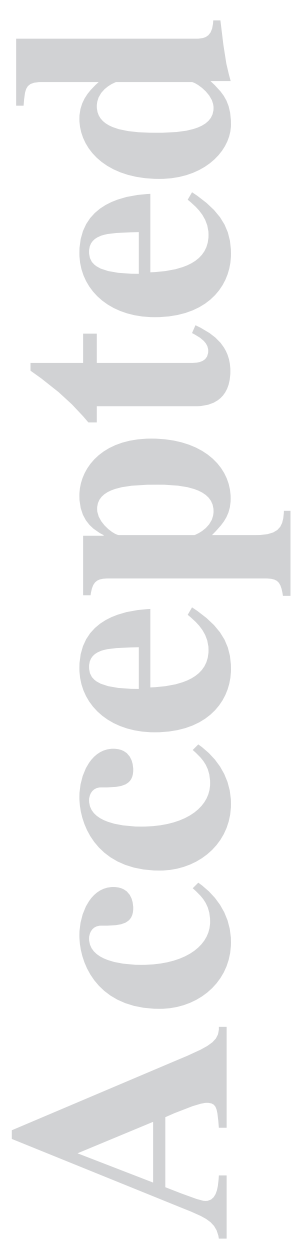




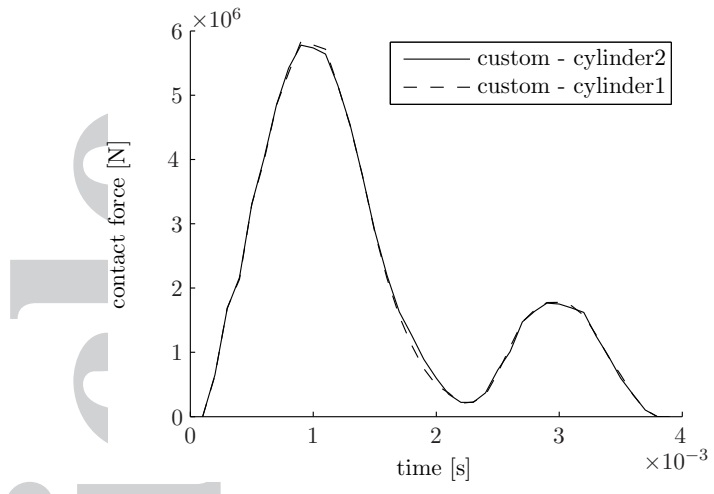

(a)

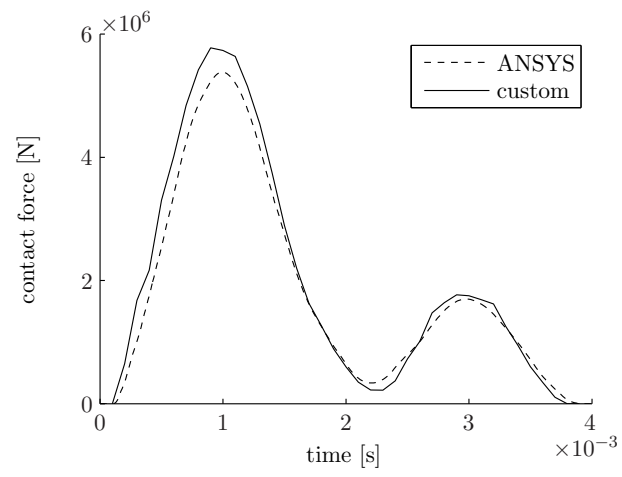

(b)

Figure 7. Magnitude of the contact force computed from the summation of all nodal contact forces applied to each of the cylinders (a); comparison to the contact force calculations obtained with the commercial software ANSYS (b).
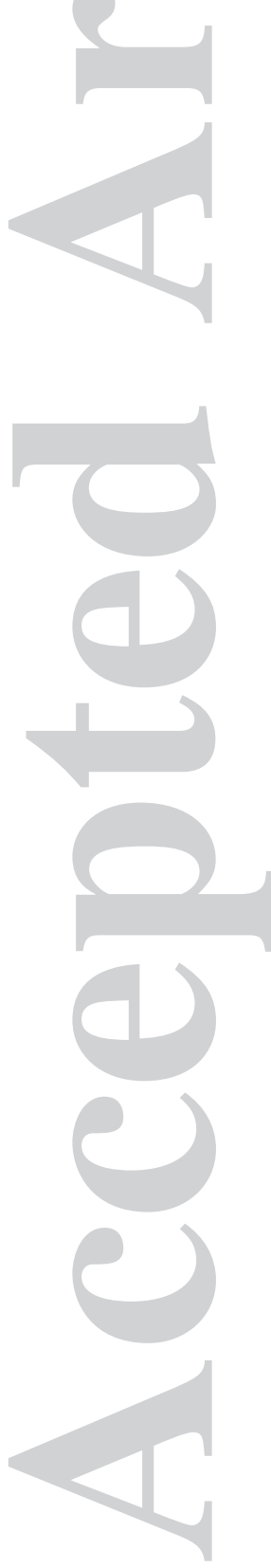

This article is protected by copyright. All rights reserved. 


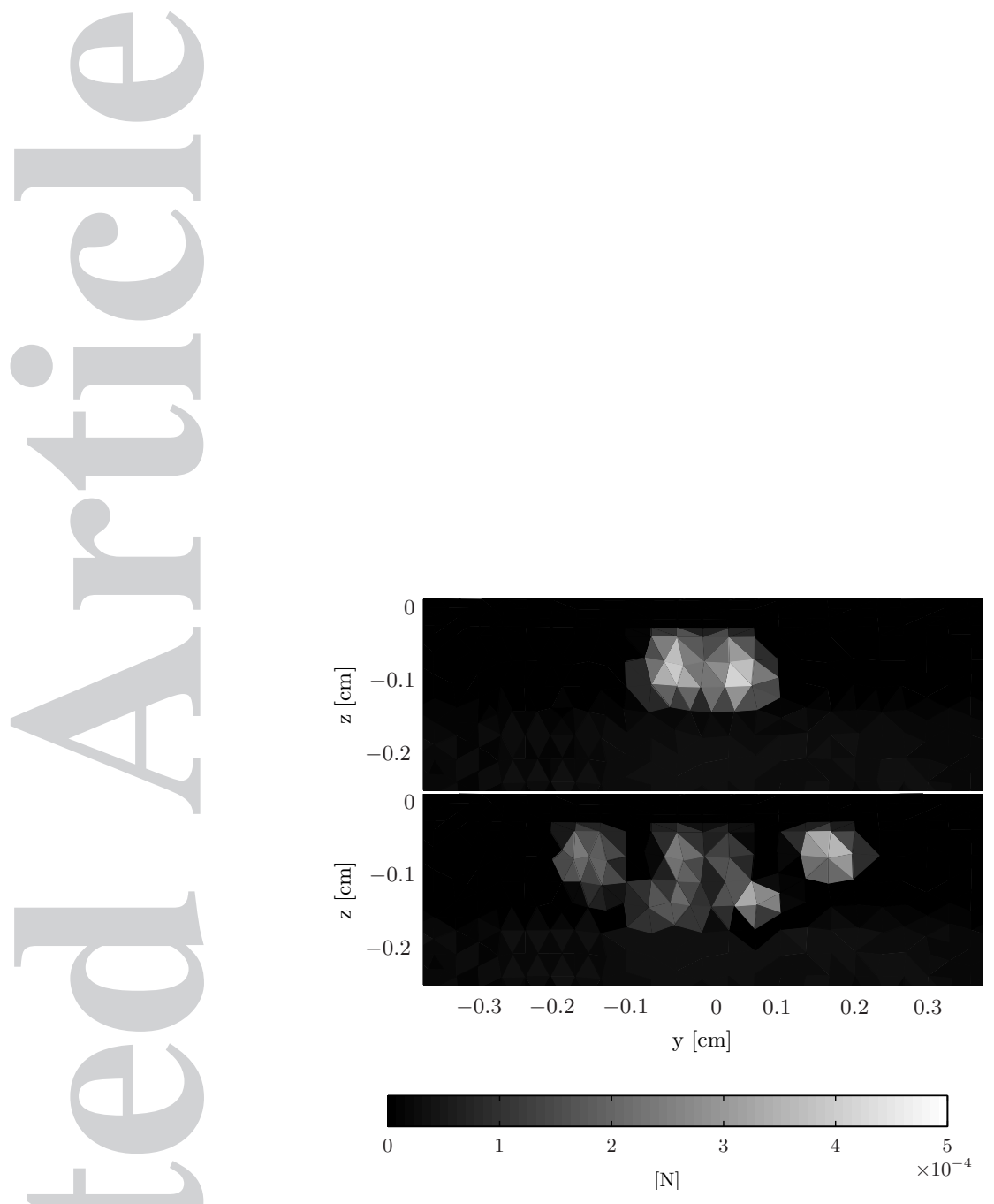

Figure 8. Sagittal view of the superficial layer of the right vocal fold $(x>0)$ during collision, from top to bottom at $t=0.0371,0.0372 \mathrm{~s}$. In grayscale, the magnitude of the $x$ component of the contact and aerodynamic forces acting on interface triangles. Downstream the lowest contact node zero aerodynamic forces are considered due to the no vocal tract coupling and the flow stop criterion defined by the zero flux parameter $\tau=0.5$ (see Section 2.4 and Table I). 

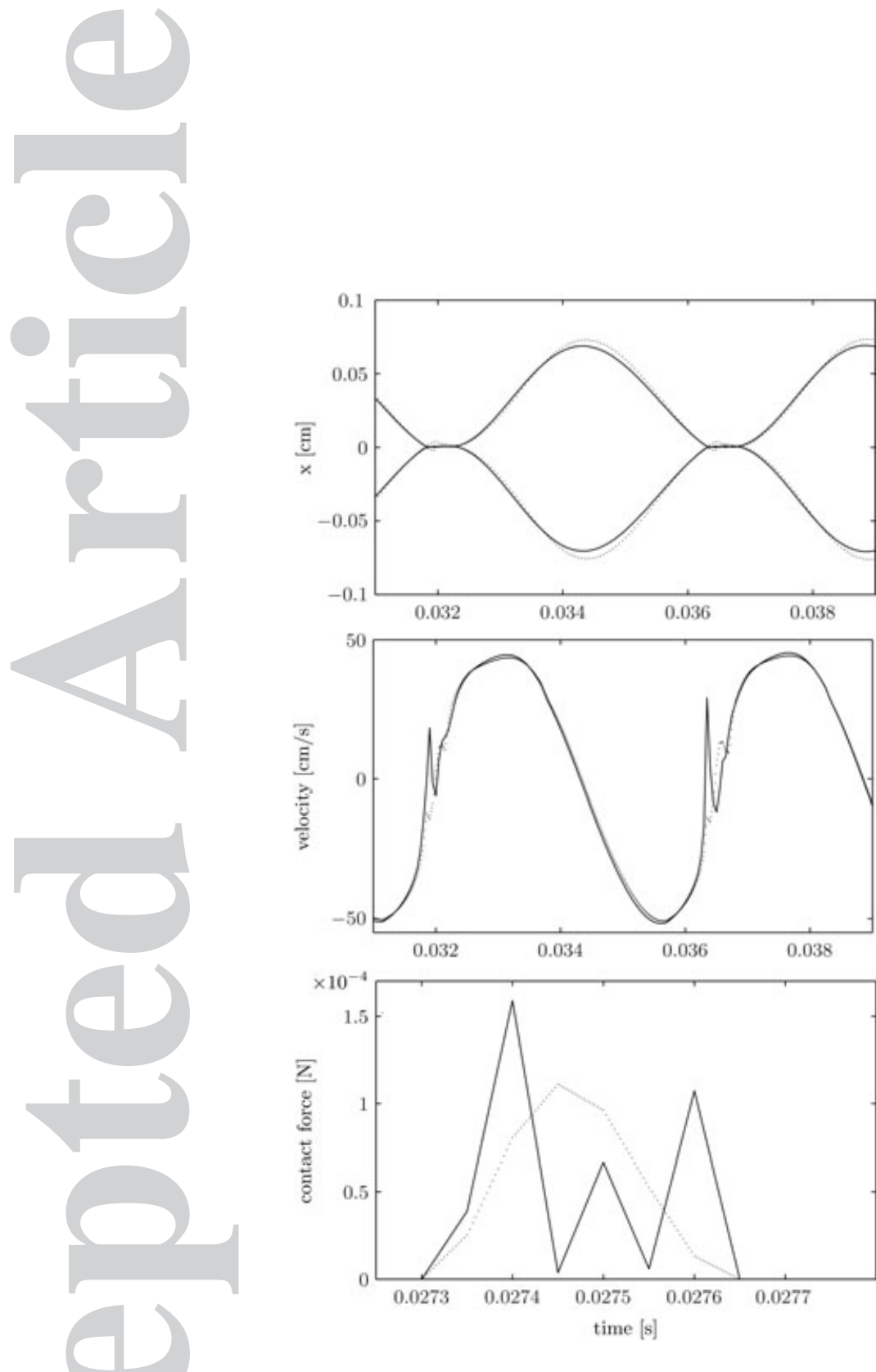

(a)

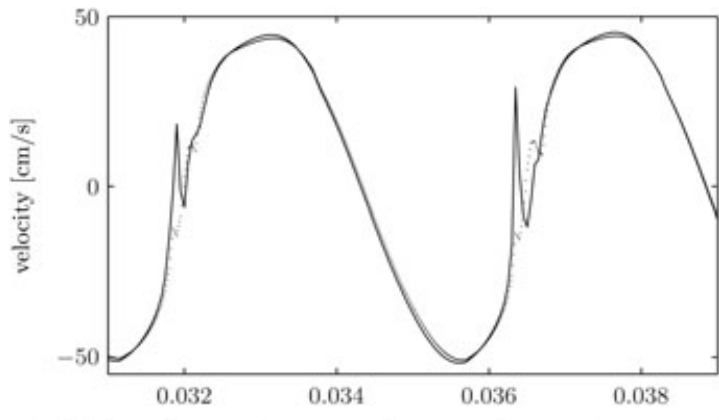

(b)

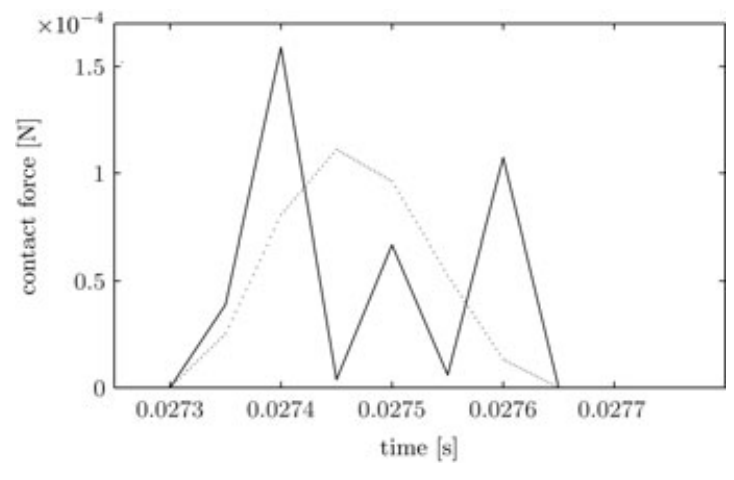

(c)

Figure 9. From top to bottom: $x$ coordinate of spatial position, velocity, and nodal contact force for an interface node at initial material coordinates $(0.024,-0.136,-0.037)$. For comparison reasons, the top figure includes the spatial position of the opposite interface nodes at material coordinates $(-0.024,-0.136,-0.037)$. Solid lines indicates the results for the Lagrange formulation; dotted lines indicate the results for a penalty formulation with $\kappa=10^{7}$. 

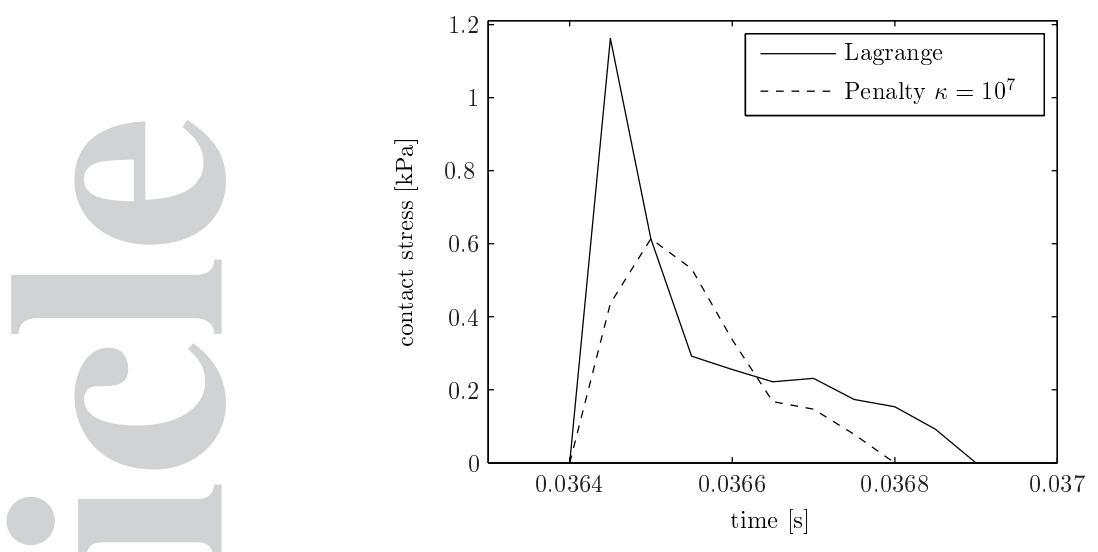

Figure 10. Mediolateral component of the contact stress with a subglottal pressure of $0.8 \mathrm{kPa}$ for a Lagrangian and a penalty $\left(\kappa=10^{7}\right)$ solution.
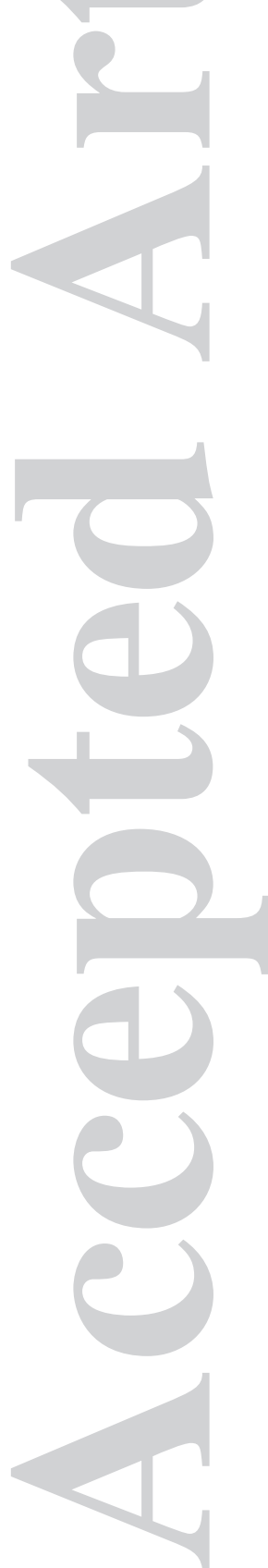

This article is protected by copyright. All rights reserved. 

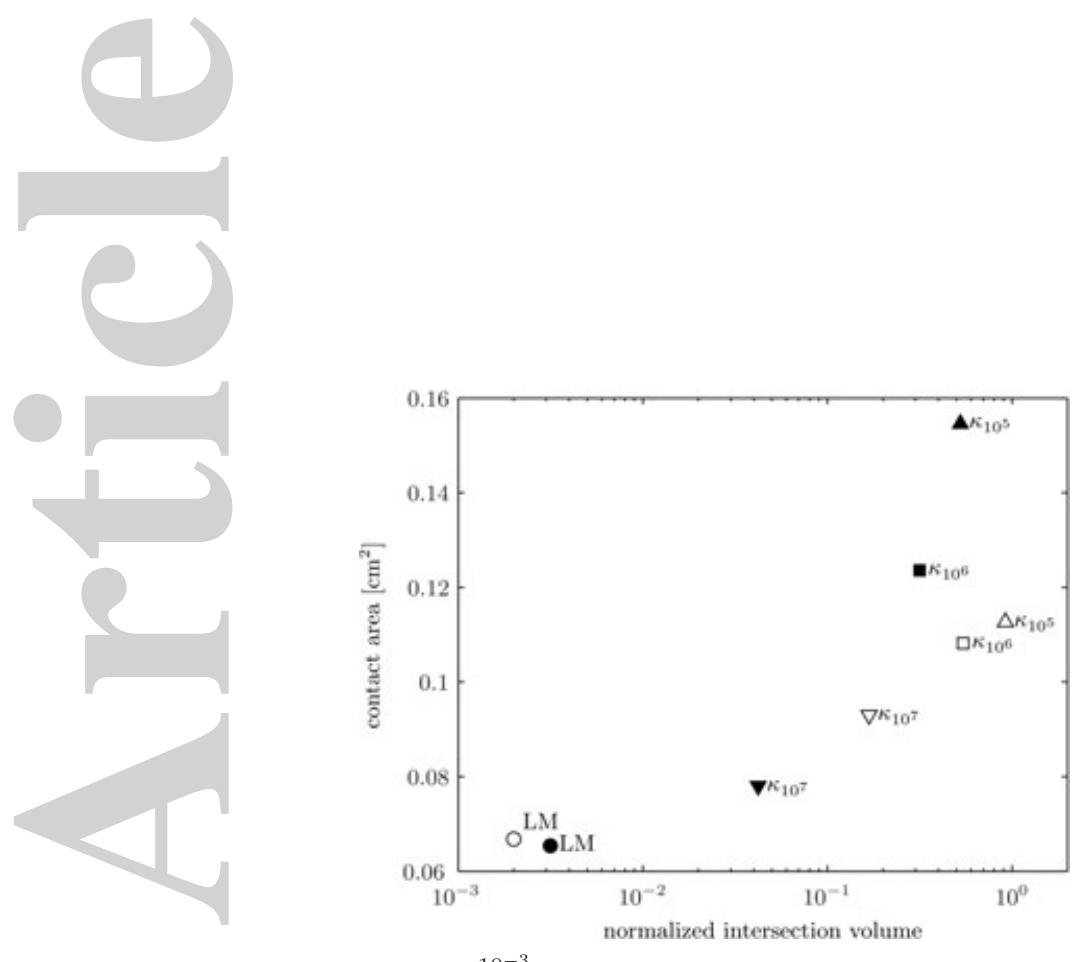

(a)
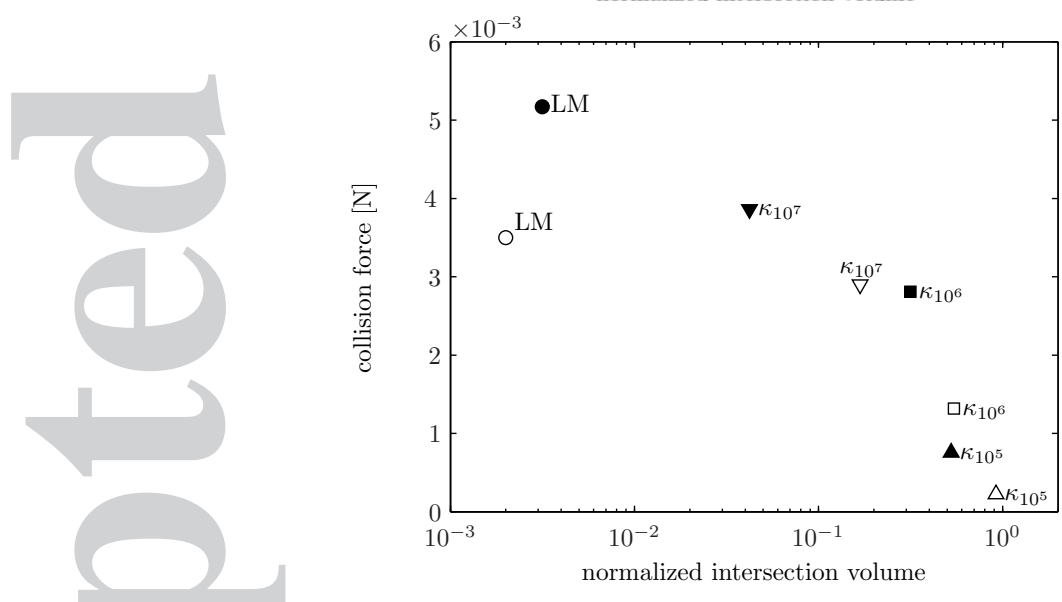

(b)

Figure 11. (a) Maximum contact area computed from the summation of interface triangles with any vertex loaded by a collision force. (b) Maximum $x$ component of the total contact force. The horizontal axis corresponds to the intersection volume normalized to the maximum intersection volume when the effect of contact forces is neglected. Circles indicate results for the Lagrange multiplier method (LM); downward triangles, squares, and upward triangles correspond to a penalty formulation with $\kappa=10^{7}, 10^{6}$ and $10^{5}$, respectively. Black and white marks indicate results for $p_{s u b}=0.8 \mathrm{kPa}$ and $p_{\text {sub }}=0.6 \mathrm{kPa}$, respectively. The selected collision time interval is [0.036, 0.037], and the right vocal fold $(x>0)$ has been used for calculations. 


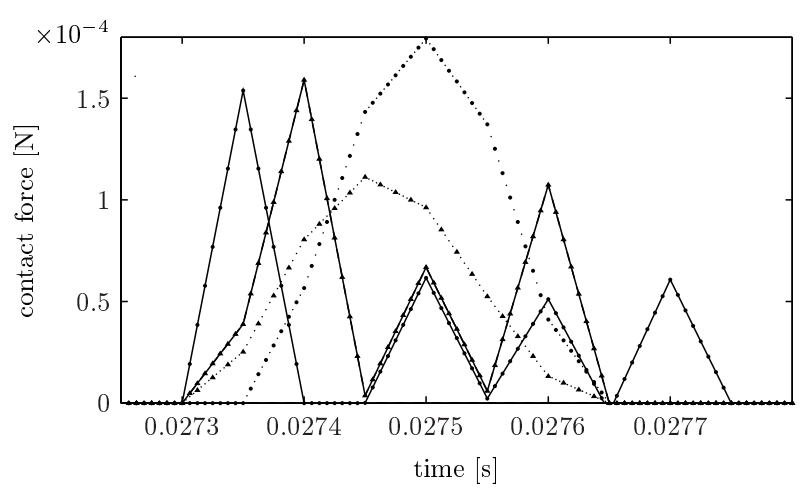

Figure 12. Contact force of a interface node at initial material coordinates $(0.024,-0.136,-0.037)$, as in Figure 9c. Circles correspond to an undamped Newmark with $\gamma=\frac{1}{2}$, and triangles to the $\alpha$-method with $\alpha=-0.3$. Dotted line indicates a penalty method with $\kappa=10^{7}$, and the solid line, the Lagrange multiplier method, as in Figure 9. Time step is $h=50 \mu \mathrm{s}$. 


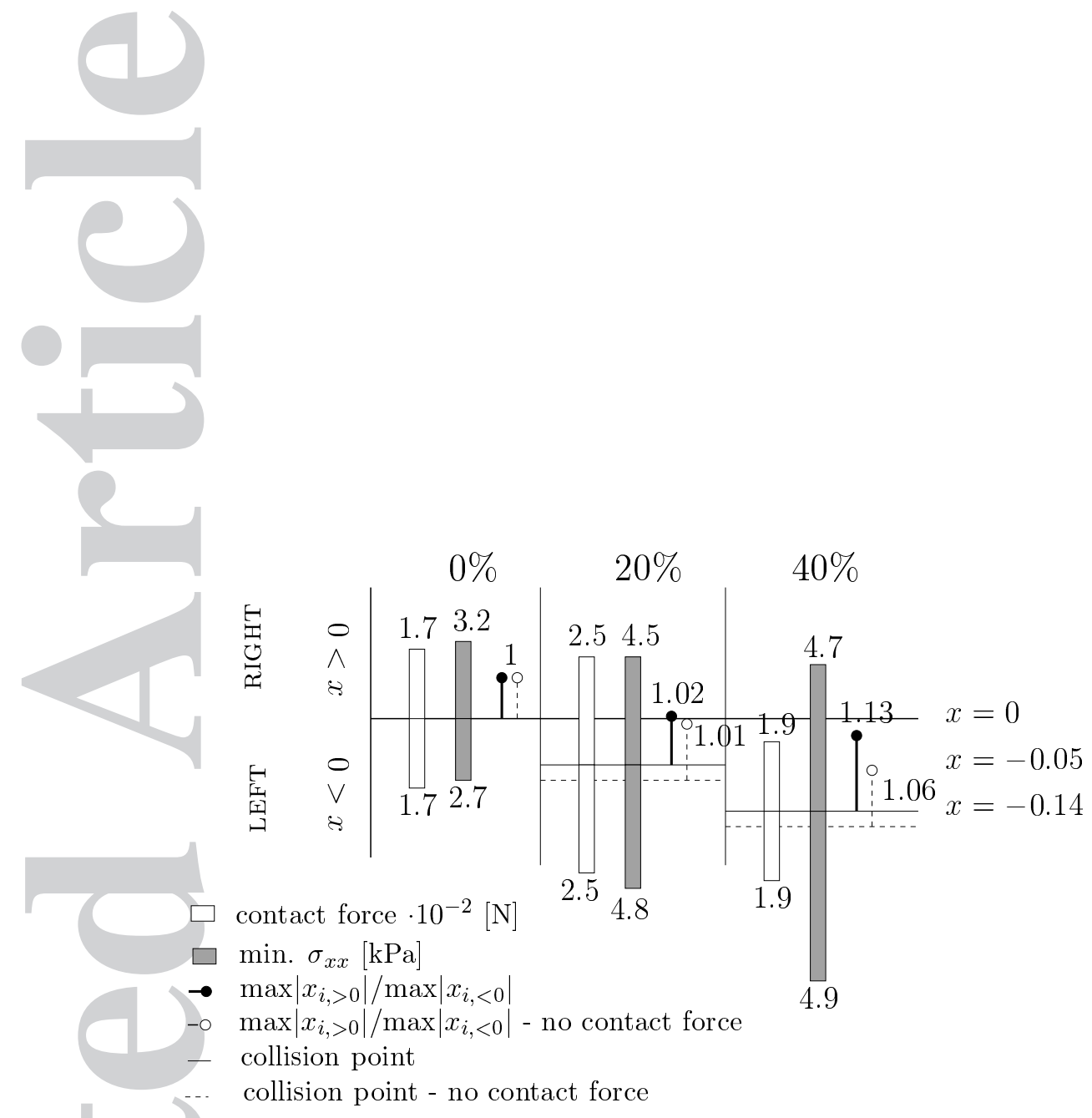

Figure 13. For each vocal fold, total contact force in $\mathrm{N}$ (white bar), maximum compressive stress in $\mathrm{kPa}$ (gray bar) and ratio between right and left vocal fold vibration amplitude (dot clip) for 0\%, $20 \%$ and $40 \%$ longitudinal Young's modulus mismatch of the cover layer. Solid horizontal line indicates the $x$ spatial coordinate of the collision point of the node 6157 , normalized to the initial material coordinate. Dashed lines indicate that the effect of surface contact forces is neglected. Values are obtained from the average over 4 cycles after transient oscillations. 


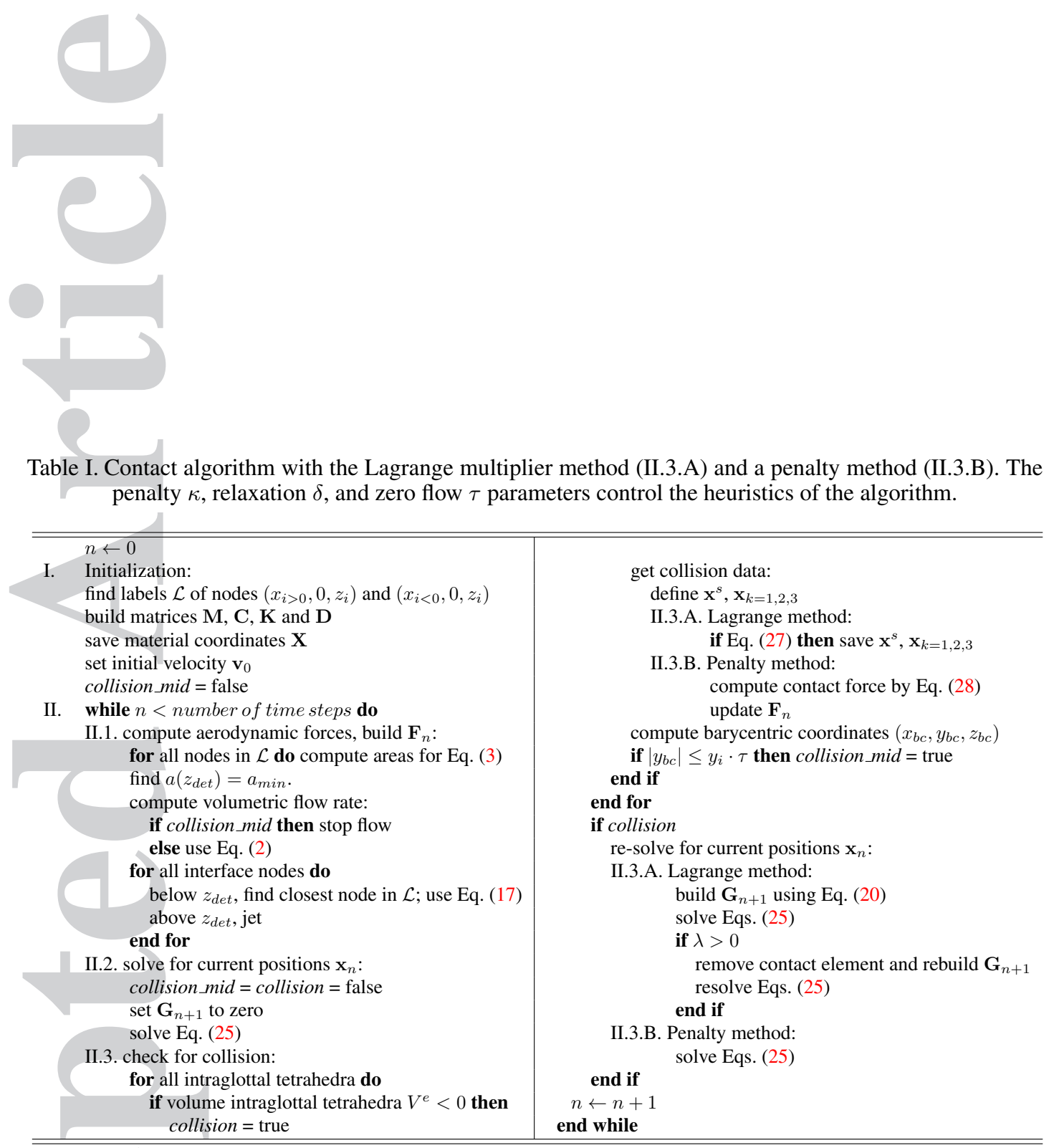

This article is protected by copyright. All rights reserved. 
Table II. Larynx geometry adapted from [17], parallel symmetric prephonatory intraglottal profile (in $\mathrm{cm}$ ). Spatial discretization of the vocal fold boundary assumes 51 elliptical shaped cross-sections; see Figure 1 and Eq. (3). For the sake of brevity, some relevant sections are shown. $y_{i}=0.7 \mathrm{~cm}$ for all $i$, and cover and ligament has a thickness of $0.05 \mathrm{~cm}$ between section $S_{17}$ and $S_{27}$.

\begin{tabular}{l|cc||c|cc}
\hline \hline & $z_{i}$ & $\left|x_{i,<}\right|=\left|x_{i,>}\right|$ & & $z_{i}$ & $\left|x_{i,<}\right|=\left|x_{i,>}\right|$ \\
\hline$S_{0}$ & -2 & 0.7 & $\vdots$ & & \\
$S_{7}$ & -0.78 & 0.6 & $S_{26}$ & -0.04 & 0.025 \\
$S_{8}$ & -0.45 & 0.25 & $S_{27}$ & -0.02 & -0.05 \\
$S_{12}$ & -0.42 & 0.16 & $S_{36}$ & 0.03 & 0.3 \\
$S_{17}$ & -0.35 & 0.05 & $S_{43}$ & 0.11 & 0.5 \\
$S_{18}$ & -0.31 & 0.025 & $S_{51}$ & 2 & 0.7 \\
\hline \hline
\end{tabular}
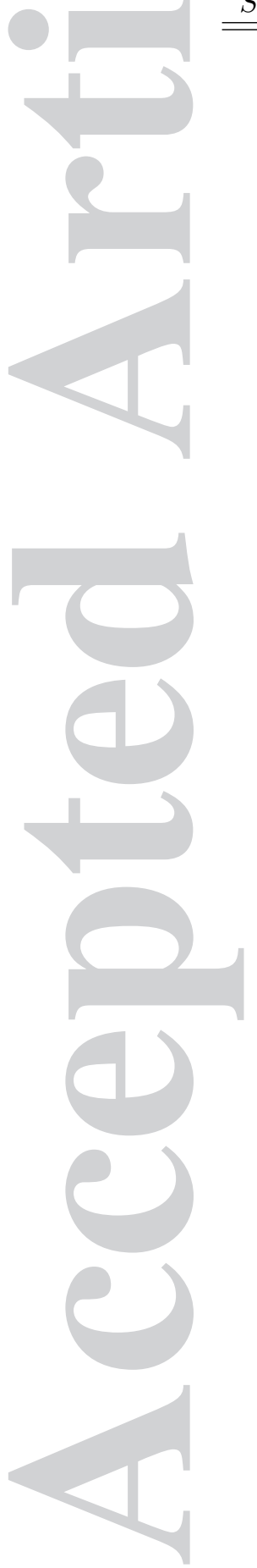
Table III. Tissue parameters used in the finite element simulation for a normal larynx, adapted from [17]. $(E, \nu)$, transversal Young's modulus and Poisson's ratio; $\left(E^{\prime}, \mu^{\prime}, \nu^{\prime}\right)$, longitudinal Young's and shear modulus, and Poisson's ratio. $\eta$ indicates viscosity. All values are specified in the centimeter-gram-second system of units.

\begin{tabular}{lcccccc}
\hline \hline & $E$ & $\nu$ & $E^{\prime}$ & $\mu^{\prime}$ & $\nu^{\prime}$ & $\eta$ \\
\hline Cover & $1 \cdot 10^{4}$ & 0.66 & $10 \cdot 10^{4}$ & $10 \cdot 10^{4}$ & 0.66 & 4 \\
Ligament & $3 \cdot 10^{4}$ & 0.58 & $20 \cdot 10^{4}$ & $40 \cdot 10^{4}$ & 0.58 & 10 \\
Body & $20 \cdot 10^{4}$ & 0.45 & $40 \cdot 10^{4}$ & $30 \cdot 10^{4}$ & 0.45 & 10 \\
\hline
\end{tabular}
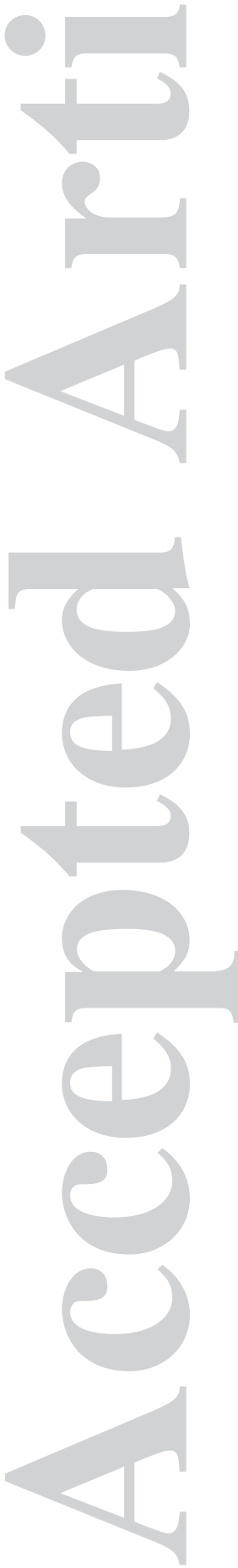

This article is protected by copyright. All rights reserved. 
Table IV. Peak mediolateral contact stress for different subglottal pressures. For comparison reason, units are in $\mathrm{kPa}$ [33].

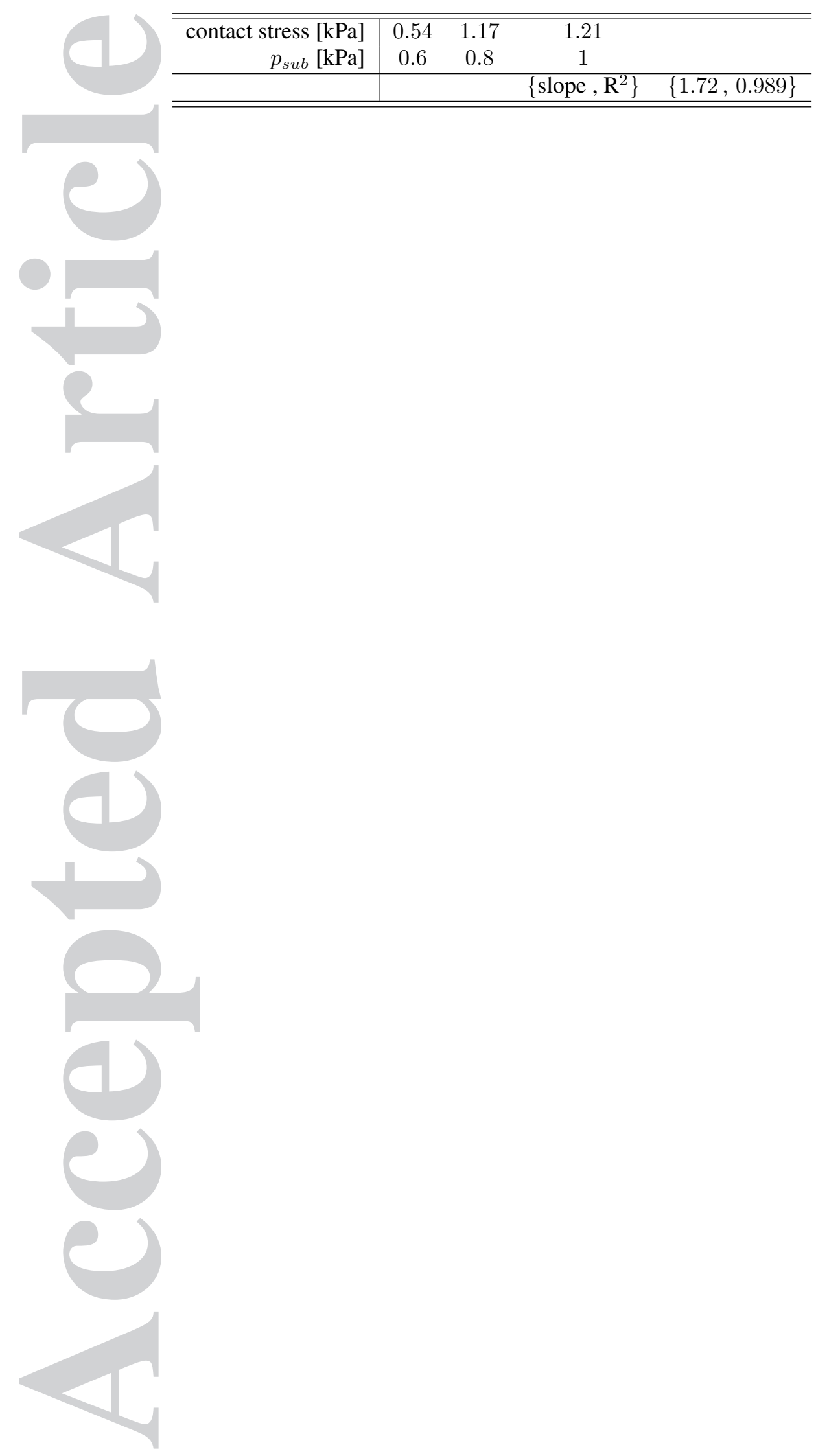

This article is protected by copyright. All rights reserved. 